\title{
Cache-aided Simultaneous Wireless Information and Power Transfer (SWIPT) with Relay Selection
}

\author{
Sumit Gautam, Student Member, IEEE, Thang X. Vu, Member, IEEE, Symeon Chatzinotas, \\ Senior Member, IEEE, and Björn Ottersten, Fellow, IEEE.
}

\begin{abstract}
In this paper, we investigate the performance of cache-assisted simultaneous wireless information and power transfer (SWIPT) cooperative systems, in which one source communicates with one destination via the aid of multiple relays. In order to prolong the relays' serving time, the relays are assumed to be equipped with a cache memory and energy harvesting (EH) capability. Based on the time-splitting mechanism, we analyze the effect of caching on the system performance in terms of the serving throughput and the stored energy at the relay. In particular, two optimization problems are formulated to maximize the relay-destination throughput and the energy stored at the relay subject to some quality-of-service (QoS) constraints, respectively. By using the KKT conditions and with the help of the Lambert function, closed-form solutions are obtained for the two formulated problems. In order to further improve the performance, a relay selection policy is introduced to select the best relay based on either the maximum throughput between the relays' and destination link or maximum stored energy at the relay, for conveying information to the destination. Numerical results reveal significant benefits of incorporating caching capabilities to SWIPT systems, in terms of improved serving time, throughput and energy harvesting performance at the relays.
\end{abstract}

Index Terms-Simultaneous wireless information and power transfer, relay systems, wireless caching, energy harvesting, relay selection.

\section{INTRODUCTION}

$\mathbf{T}$ HE exponential increase in the usage of wireless devices like smart-phones, wearable gadgets, or connected vehicles, has not only posed substantial challenges to meet the performance and capacity demands [1], [2], but also revealed some serious environmental concerns with alarming energy consumption and $\mathrm{CO}_{2}$ emissions [3]. These concerns become more significant as the forecast number of devices will exceed 50 billions by the end of 2020 [4]. Recent developments in the upcoming paradigm of Internet-of-Things (IoT) emphasize the interconnection between equipments, commodities, functionalities, and customers, with or without human mediation. Since most of these connecting operations involve wireless sensor nodes or equivalent battery-limited devices that may not be continuously powered, energy becomes a sparse and pivotal resource. These challenges require the future communication

The authors are with the Interdisciplinary Centre for Security, Reliability and Trust (SnT), University of Luxembourg, L-1855 Luxembourg. E-mail: \{sumit.gautam, thang.vu, symeon.chatzinotas, bjorn.ottersten\}@uni.lu.

Part of this work has been published in IEEE Wireless Communications and Networking Conference 2018 (WCNC'18).

This research work is financially supported, in part, by the Luxembourg National Research Fund under bilateral project FNR-FNRS InWIP-NET and project FNR CORE ProCAST, and by the European Research Council under project AGNOSTIC. networks to have not only efficient energy management but also capability of being self-powered from redundant energy sources, which is known as energy harvesting $(\mathrm{EH})$.

Among potential EH techniques, simultaneous wireless information and power transfer (SWIPT) has received much attention as the key enabling technique for future IoT networks [5]. The basic premise behind SWIPT is to allow concurrent data reception and $\mathrm{EH}$ from the same radio frequency (RF) input signal. Considering rapid drainage of battery sources in wireless devices, it has almost become essential to take up such techniques in order to compensate for this issue. Since the conventional receiver architectures are capable of performing information decoding with focus on increasing the data rate only and are unable to harvest energy, this calls for alternative receiver architectures to support SWIPT [6]. Two notable architectures have been proposed based on time switching (TS) and power splitting (PS) schemes [7], [8]. In the former, the received signal is switched between the information decoder and energy harvester. It is noted in the TS scheme that full received power is used for either information decoding or energy harvesting. In the latter, both the information decoder and energy harvester are active simultaneously, each of them receive parts of the signal power. The research on SWIPT has received much attention recently [9]-[11]. The Rate-Energy (R-E) trade-off is analyzed in [12] under both TS and PS based SWIPT. The authors in [13] investigate the performance of SWIPT ad-hoc networks in terms of transmission capacity and the harvested energy per unit area. The harvested energy density can be maximized by optimizing the density of the deployed information decoding nodes in TS architecture, and the power splitting ratio in PS architecture. In [14], a tractable model of the rectifier non-linearity is developed to support general multi-carrier modulated input waveforms, which is then used to model a wireless information and power transfer architecture based on the superposition of multi-carrier modulated and unmodulated waveforms. The authors in [15] propose an adaptive scheduling scheme for $\mathrm{EH}$ based multiuser systems to jointly maximize achievable rate and harvested energy. In [16], the performance of cooperative networks with battery-powered EH relays is analyzed considering direct source-relay link. The authors in [17] have investigated various relaying protocols to realize SWIPT in a dual-hop system under static (equal) time distribution. A comprehensive review on SWIPT is presented in [18].

Another major problem the future networks have to face is network congestion, which usually occurs during peak hours when the network resource is scarce. The cause of 


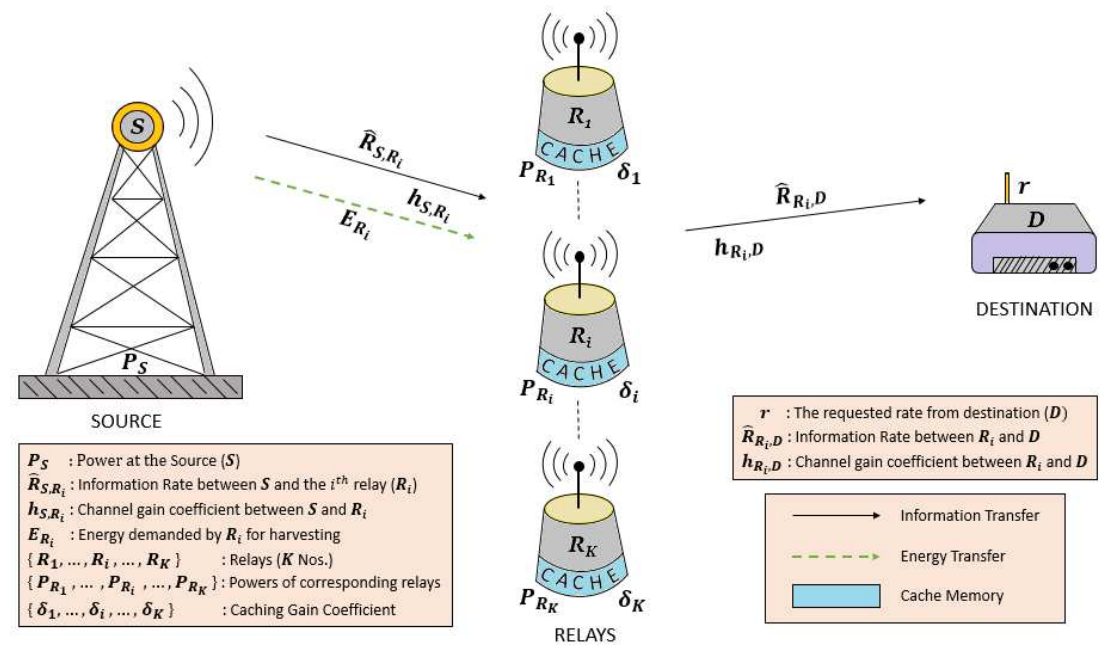

Fig. 1: System model for simultaneous wireless information and power transfer (SWIPT) with caching.

this congestion is mainly due to the fact that replicas of a common content may be demanded by various mobile users. A promising solution to overcome network congestion is to shift the network traffic from peak hours to off-peak times via content placement or caching [19]. In (off-line) caching, there is usually a placement phase and a delivery phase. In the placement phase, which usually occurs during off-peak times when the network resources are abundant, popular content is prefetched in distributed caches close to the end users. The latter usually occurs during peak hours when the actual users' requests are revealed. If the requested content is available in the user's local storage, it can be served without being requested from the core network. Various advantages brought by caching have been observed in terms of backhaul's load reduction [19]-[21] and system energy efficiency improvement [22]-[24].

A generic concept outlining joint $\mathrm{EH}$ and caching has recently been proposed for $5 \mathrm{G}$ networks to exploit the benefits of both techniques. A so-called framework GreenDelivery is proposed in [3], which enables efficient content delivery in small cells based on energy harvesting small base stations. In [25], an online energy-efficient power control scheme for EH with caching capability is developed. In particular, by adopting the Poisson distribution for the energy sources, a dynamic programming problem is formulated and solved iteratively by using numerical methods. The authors in [26] propose a caching mechanism at the gateway for the energy harvesting based IoT sensing service to maximize the hit rate. In [27], the authors investigate the performance of heterogeneous vehicular networks with renewable energy source. A network planning problem is formulated to optimize cache size and energy harvesting rate subject to backhaul capacity limits. We note that these works either address an abstract EH with general external energy sources or consider EH separated from caching.

On one hand, the SWIPT-based systems are inevitably impacted by the phenomenon of R-E trade-off [28]. On the other hand, cooperative systems with incorporated caches are proven to perform far better in terms of information exchange compared to traditional communication systems without caching [29]. In this paper, we intend to develop a joint relationship between caching and SWIPT to study the corresponding performance benefits. Besides, significant benefits are anticipated from cache-assisted SWIPT systems, in terms of: (i) prolonged serving time of the relays, (ii) reduced content delivery time, (iii) increased transmission throughput, and (iv) improved energy storage at the relay (including energy harvesting capabilities). In this regard, we investigate the performance of EH based cooperative networks, in which the relay node is equipped with both SWIPT and caching capabilities. In particular, we aim at developing a framework to realize the integration of SWIPT with caching architectures. The considered system is assumed to operate in the TS based mode, since the PS counterpart imposes complex hardware design challenges of the power splitter [8]. The main contributions of this paper are four-fold, listed as follows.

1) Firstly, we introduce a novel cache-assisted SWIPT architecture for decode-and-forward (DF)-enabled relaying systems under a dynamic TS-based scheme to study the interaction between caching capacity and SWIPT in the considered system.

2) Secondly, an optimization problem is formulated to maximize the throughput of the (serving) link between the relay and destination, taking into account the caching capacity, minimum harvested energy and quality-ofservice (QoS) constraints. By using the KKT conditions with the aid of the Lambert function, a closed-form solution of the formulated problem is obtained for the dynamic TS factors and the transmit power at the relay. Based on this result, the best relay will be selected for cooperation.

3) Thirdly, we formulate an optimization problem to maximize the energy stored at the relay subjected to the QoS constraint. Similar to the previous problem, a closed-form solution is obtained for optimization of the dynamic TS factors and the transmit power at the relay, by using the KKT conditions and the Lambert function.

4) Finally, the effectiveness of the proposed schemes are demonstrated via intensive numerical results, through which the impacts of key system parameters are observed. Furthermore, a discussion on practical implementation and future directions is provided.

The remainder of this paper is organized as follows. Sec- 


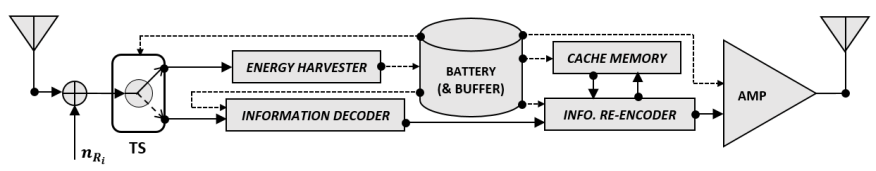

Fig. 2: Proposed DF relay transceiver design for hybrid SWIPT and caching with time switching (TS) architecture.

tion III describes the system model and relevant variables. Section III presents the problem for maximization of link throughput between the relay and destination. Section IV maximizes the stored energy at the relay. In Section $V$ numerical results are presented to demonstrate the effectiveness of the proposed architectures. Finally, Section VI concludes the paper.

\section{SySTEM MODEL}

We consider a generic TS based SWIPT system, which consists of one source, $K$ relays, and one destination, as depicted in Fig. 1. Each device within the network is equipped with single antenna. Due to limited coverage, e.g., transmit power limit or blockage, there is no direct connection between the source and the destination. The considered model can find application on the downlink where the base station plays the source's role and sends information to a far user via a smallor femto- cell base station. The relays operate in DF mode and are equipped with single antenna. We consider a general cache-aided SWIPT model, in which each relay contains an information decoder, an energy harvester, and a cache in order to store or exchange information. The block diagram of a typical relay architecture is shown in Fig. 2

We consider block Rayleigh fading channels, in which the channel coefficients remain constant within a block (or coherence time), and independently change block to block. One communication session (broadcasting and relaying) takes place in $T$ seconds ( $T$ does not exceed the coherence time). In the broadcasting phase, the source-relay links are active for information decoding (at the relay) and energy harvesting. The relays employ the TS scheme so that the received signal is first provided to the the energy harvester for some fraction of the time allocated for transmitter-relay communication link, and then to information decoder for the remaining fraction. In case of equal time slots in both the hops, this mechanism is also known as harvest-then-forward protocol [30]. Correspondingly, due to the changing nature of the TS factors', we refer to this analogous operation as "dynamic harvest-thenforward" protocol. In the relaying phase, the selected relay forwards the information to the destination. Full channel state information (CSI) is assumed to be available at a centralized base station which performs all the computations and inform the relevant devices via adequate signaling. The relay with the best reward will be selected for sending information to the destination. Details on relay selection will be presented later.

Fig. 3 presents a convention for allocation of time fractions in the TS scheme: i) energy transmission from the source to $i$-th relay, ii) information processing at the relay, and iii) information forwarding to the destination. The link between the transmitter and $i$-th relay with cache is active for a fraction of $\beta_{i} T$ seconds, while the link between the $i$-th relay and destination is active for the remaining $\left(1-\beta_{i}\right) T$,

\begin{tabular}{|c|c|c|}
\hline$\alpha_{i} \beta_{i} T$ & $\left(1-\alpha_{i}\right) \beta_{i} T$ & $\left(1-\beta_{i}\right) T$ \\
\hline $\begin{array}{l}\text { Energy Transmission } \\
\text { from Source to } i \text {-th Relay }\end{array}$ & $\begin{array}{l}\text { Information Transmission } \\
\text { from Source to } i \text {-th Relay, } \\
\text { Caching, and Re-encoding }\end{array}$ & $\begin{array}{l}\text { Information Transmission } \\
\text { from } i \text {-th Relay to the Destination }\end{array}$ \\
\hline
\end{tabular}

Fig. 3: Convention assumed for distribution of time to investigate the throughput maximization problem.

where $0 \leq \beta_{i} \leq 1$. Furthermore, we assume that the energy harvesting at the relays takes place for a fraction of $\alpha_{i} \beta_{i} T$ seconds and the information decoding at the relay takes place for a fraction of $\left(1-\alpha_{i}\right) \beta_{i} T$ seconds, where $0 \leq \alpha_{i} \leq 1$. For ease of representation, we assume normalized time to use energy and power interchangeably without loss in generality.

\section{A. Signal model}

We define the information transfer rates from the source to the $i$-th relay as $\hat{R}_{S, R_{i}}$, and from the $i$-th relay to the destination as $\hat{R}_{R_{i}, D}$, with $1 \leq i \leq K$, where $i \in \mathbb{Z}$.

Let $h_{S, R_{i}}$ and $h_{R_{i}, D}$ denote the channel coefficients between the source and the $i$-th relay and between the $i$-th relay and the destination, respectively, and $d_{S, R_{i}}$ and $d_{R_{i}, D}$ denote the distance between the source and $i$-th relay, and the distance between the $i$-th relay and destination, respectively. Furthermore, $P_{S}$ and $P_{R_{i}}$ denote the transmit power at the source and the $i$-th relay, respectively.

Let $x \in \mathbb{C}$ be the transmitted symbol by the source satisfying $E\left\{|x|^{2}\right\}=1$. The signal received at the $i$-th relay is given by

$$
y_{R_{i}}=\sqrt{P_{S}} d_{S, R_{i}}^{-\vartheta / 2} h_{S, R_{i}} x+n_{R_{i}},
$$

where $\vartheta$ is the path loss exponent, $n_{R_{i}}$ is the additive white Gaussian noise (AWGN) at the relay, which is an independent and identically distributed (i.i.d.) complex Gaussian random variable with zero mean and variance $\sigma_{n_{R_{i}}}^{2}$.

Upon receiving the desired signal from the source, the relay decodes to obtain the estimate of the original signal. Then the (selected) relay re-encodes and forwards it to the destination. The signal received at the destination as transmitted by the $i$-th relay is given by

$$
y_{D}=\sqrt{P_{R_{i}}} d_{R_{i}, D}^{-\vartheta / 2} h_{R_{i}, D} \tilde{x}+n_{D},
$$

where $\tilde{x}$ is the transmit symbol from the relay, and $n_{D}$ is the AWGN at the destination node which is an i.i.d. complex Gaussian random variable with zero mean and variance $\sigma_{n_{D}}^{2}$. We note that in the proposed cached-aid architecture, the relayed symbol can be either decoded source symbol or from the relay's cache.

The effective signal-to-noise ratio (SNR) of the source-relay and relay-destination links are given as

$$
\gamma_{S, R_{i}}=\frac{P_{S} d_{S, R_{i}}^{-\vartheta}\left|h_{S, R_{i}}\right|^{2}}{\sigma_{n_{R_{i}}}^{2}} ; \gamma_{R_{i}, D}=\frac{P_{R_{i}} d_{R_{i}, D}^{-\vartheta}\left|h_{R_{i}, D}\right|^{2}}{\sigma_{n_{D}}^{2}} \text {. }
$$

By assuming a Gaussian codebook, the achievable information rate on the source-relays' link is

$$
\hat{R}_{S, R_{i}}=B \log _{2}\left(1+\gamma_{S, R_{i}}\right), \quad \forall i
$$

and the achievable information rate at the destination is

$$
\hat{R}_{R_{i}, D}=B \log _{2}\left(1+\gamma_{R_{i}, D}\right), \forall i,
$$

where $B$ is the channel bandwidth. 


\section{B. Caching model}

A general caching model is considered at the relays. In particular, in addition to the information sent from the source, the relays have access to the information stored in their individual caches to serve the destination. For robustness, we assume that for $i=1, \ldots, K$, the $i$-th relay does not have information about the content popularity. Therefore, it will store $0 \leq \delta_{i} \leq 1$ parts of every file in its cache [19], [22] $]^{1}$. For convenience, we call $\delta_{i}$ as the caching coefficient throughout the paper. This caching scheme will serve as the lower bound benchmark compared to the case where priori information of content popularity is available. When the destination requests a file from the library, $\delta_{i}$ parts of that file are already available at the $i$-th relay's cache. Therefore, the source need to send only the remaining portions of that file to the relay.

\section{Power assumption at the relay}

For robustness, we assume that the relays are powered by an external source, $E_{\text {ext }}$, on top of the harvested energy. This general model allows to analyze the impact of various practical scenarios. As an usecase, the purely SWIPT relay is obtained by setting $E_{\text {ext }}$ to zero. The harvested energy at the $i$-th relay is given by

$$
E_{R_{i}}=\zeta \alpha_{i} \beta_{i}\left(P_{S} d_{S, R_{i}}^{-\vartheta}\left|h_{S, R_{i}}\right|^{2}+\sigma_{n_{R_{i}}}^{2}\right),
$$

where $\zeta$ is the energy conversion efficiency of the receiver.

\section{MAXIMIZATION OF THE SERVING INFORMATION RATE}

In this section, we aim at maximizing the serving information data between the selected relay and the destination, by taking into consideration the caching capacity at the relay, while assuring the predefined QoS constraint and that the total transmit power at the source does not exceed the limit. The corresponding optimization problem (P1) is stated as follows

$$
\begin{aligned}
(P 1): \max _{i \in \mathcal{K}, \alpha_{i}, \beta_{i}, P_{R_{i}}} & \left(1-\beta_{i}\right) \hat{R}_{R_{i}, D} \\
\text { subject to : } & (C 1):\left(1-\alpha_{i}\right) \beta_{i}\left(\hat{R}_{S, R_{i}}+\left(\delta_{i} \cdot r\right)\right) \\
& \geq\left(1-\beta_{i}\right) \hat{R}_{R_{i}, D} \\
& (C 2):\left(1-\beta_{i}\right) P_{R_{i}} \leq E_{R_{i}}+E_{\text {ext }} \\
& \\
& (C 3): 0<P_{S} \leq P^{\star} \\
& (C 4): 0 \leq \alpha_{i} \leq 1 \\
& (C 5): 0 \leq \beta_{i} \leq 1
\end{aligned}
$$

where $\mathcal{K}=\{1, \ldots, K\}, E_{R_{i}}$ is given in [6, $E_{\text {ext }}$ is the external energy required at the relay for further transmission of the signal, $P^{\star}$ is the maximum power limit at the transmitter, and $r$ is the QoS constraint. The objective in (7) is to maximize the transfered data to the destination, since the relaydestination link is active only in $1-\beta_{i}$ (active times normalized by $T)$. Constraint $(7 \mathrm{a})$ is to assure non-empty buffer at the relay. Constraint (7b) is to assure that the used energy at the relay cannot exceed the input. It is noteworthy that $E_{\text {ext }}$ is stipulated in each time slot which thereby translates into

\footnotetext{
${ }^{1}$ This caching method is also known as probabilistic caching.
}

stored energy for the successive time slot, provided the relays are battery driven. In other case, $E_{e x t}$ can be assumed to be constant in each time slot if the relays are part of the infrastructure with sufficient power supply, as in [31].

This is a mixed-integer programming problem implying that relay selection along with joint computations of $\alpha_{i}, \beta_{i}$, and $P_{R_{i}}$ is a difficult task. Therefore, we recast $(P 1)$ into a pair of coupled optimization problems namely, outer optimization for choosing the best relay, and inner optimization for joint computations of $\alpha_{i}, \beta_{i}$, and $P_{R_{i}}$. In the following sections, we address the optimal solutions to the inner and outer optimizations, respectively.

\section{A. Optimization of TS Factors and the Relay Transmit Power}

In this subsection, we address the inner optimization problem of (P1) involving joint computations of $\alpha_{i}, \beta_{i}$, and $P_{R_{i}}$, assuming that the $i$-th relay is active. The consequent subproblem is formulated as follows

$$
\begin{aligned}
(P 2): \max _{\alpha_{i}, \beta_{i}, P_{R_{i}}} & \left(1-\beta_{i}\right) \hat{R}_{R_{i}, D} \\
\text { subject to : } & 7 \mathrm{a}-7 \mathrm{e} .
\end{aligned}
$$

This is a non-linear programming problem involving joint computations of $\alpha_{i}, \beta_{i}$, and $P_{R_{i}}$, which is challenging to find the exact solution. Since the constraints are partially convex on each variable while fixing the others, we propose to solve this problem using the Karush-Kuhn-Tucker (KKT) conditions.

The Lagrangian corresponding to $(P 2)$ can be denoted as follows

$$
\begin{aligned}
& \mathcal{L}\left(\alpha_{i}, \beta_{i}, P_{R_{i}} ; \lambda_{1}, \lambda_{2}, \lambda_{3}, \lambda_{4}\right)=F\left(\alpha_{i}, \beta_{i}, P_{R_{i}}\right) \\
& -\lambda_{1} G\left(\alpha_{i}, \beta_{i}, P_{R_{i}}\right)-\lambda_{2} H\left(\alpha_{i}, \beta_{i}, P_{R_{i}}\right) \\
& -\lambda_{3} I\left(\alpha_{i}, \beta_{i}, P_{R_{i}}\right)-\lambda_{4} J\left(\alpha_{i}, \beta_{i}, P_{R_{i}}\right),
\end{aligned}
$$

where

$$
\begin{aligned}
& F\left(\alpha_{i}, \beta_{i}, P_{R_{i}}\right)=\left(1-\beta_{i}\right) B \log _{2}\left(1+\gamma_{R_{i}, D}\right), \\
& G\left(\alpha_{i}, \beta_{i}, P_{R_{i}}\right)=\left(1-\beta_{i}\right) B \log _{2}\left(1+\gamma_{R_{i}, D}\right) \\
& -\left(1-\alpha_{i}\right) \beta_{i}\left[B \log _{2}\left(1+\gamma_{S, R_{i}}\right)+\left(\delta_{i} \cdot r\right)\right] \leq 0, \\
& H\left(\alpha_{i}, \beta_{i}, P_{R_{i}}\right)=\left(1-\beta_{i}\right) P_{R_{i}}-\zeta \alpha_{i} \beta_{i}\left(P_{S} d_{S, R_{i}}^{-\vartheta}\left|h_{S, R_{i}}\right|^{2}\right. \\
& \left.+\sigma_{n_{R_{i}}}^{2}\right)-E_{\text {ext }} \leq 0 \text {, } \\
& I\left(\alpha_{i}, \beta_{i}, P_{R_{i}}\right)=\alpha_{i}-1 \leq 0, \\
& J\left(\alpha_{i}, \beta_{i}, P_{R_{i}}\right)=\beta_{i}-1 \leq 0 .
\end{aligned}
$$

For (local) optimality $2^{2}$ it must hold $\nabla \mathcal{L}\left(\alpha_{i}, \beta_{i}, P_{R_{i}} ; \lambda_{1}, \lambda_{2}, \lambda_{3}, \lambda_{4}\right)=0$. Thus, we can represent the equations for satisfying the optimality conditions as

$$
\begin{aligned}
\frac{\partial \mathcal{L}\left(\alpha_{i}, \beta_{i}, P_{R_{i}} ; \lambda_{1}, \lambda_{2}, \lambda_{3}, \lambda_{4}\right)}{\partial \alpha_{i}} & \Longrightarrow-\lambda_{1}\left(\beta _ { i } \left(B \log _{2}(1+\right.\right. \\
\left.\left.\left.\gamma_{S, R_{i}}\right)+\left(\delta_{i} \cdot r\right)\right)\right)-\lambda_{2}(- & \zeta \beta_{i}\left(P_{S} d_{S, R_{i}}^{-\vartheta}\left|h_{S, R_{i}}\right|^{2}\right. \\
& \left.\left.+\sigma_{n_{R_{i}}}^{2}\right)\right)-\lambda_{3}=0
\end{aligned}
$$

${ }^{2}$ Note that a KKT point is not necessarily a local optimum, but can also be saddle point and even maximum points [32]. 


$$
\begin{gathered}
\quad \frac{\partial \mathcal{L}\left(\alpha_{i}, \beta_{i}, P_{R_{i}} ; \lambda_{1}, \lambda_{2}, \lambda_{3}, \lambda_{4}\right)}{\partial \beta_{i}} \Longrightarrow-B \log _{2}\left(1+\gamma_{R_{i}, D}\right) \\
-\lambda_{1}\left(-B \log _{2}\left(1+\gamma_{R_{i}, D}\right)-\left(1-\alpha_{i}\right)\left(B \log _{2}\left(1+\gamma_{S, R_{i}}\right)+\left(\delta_{i} \cdot r\right)\right)\right) \\
-\lambda_{2}\left(-P_{R_{i}}-\zeta \alpha_{i}\left(P_{S} d_{S, R_{i}}^{-\vartheta}\left|h_{S, R_{i}}\right|^{2}+\sigma_{n_{R_{i}}}^{2}\right)\right)-\lambda_{4}=0,
\end{gathered}
$$$$
\frac{\partial \mathcal{L}\left(\alpha_{i}, \beta_{i}, P_{R_{i}} ; \lambda_{1}, \lambda_{2}, \lambda_{3}, \lambda_{4}\right)}{\partial P_{R_{i}}} \Longrightarrow
$$$$
\frac{\ln (2) d_{R_{i}, D}^{-\vartheta}\left|h_{R_{i}, D}\right|^{2}}{\sigma_{n_{D}}^{2}+P_{R_{i}} d_{R_{i}, D}^{-\vartheta}\left|h_{R_{i}, D}\right|^{2}}
$$$$
-\lambda_{1}\left(\frac{\ln (2) d_{R_{i}, D}^{-\vartheta}\left|h_{R_{i}, D}\right|^{2}}{\sigma_{n_{D}}^{2}+P_{R_{i}} d_{R_{i}, D}^{-\vartheta}\left|h_{R_{i}, D}\right|^{2}}\right)-\lambda_{2}=0 .
$$

The conditions for feasibility are as expressed in (11), (12), (13), and (14). Complementary slackness expressions can be represented as follows

$$
\begin{gathered}
\lambda_{1} \cdot G\left(\alpha_{i}, \beta_{i}, P_{R_{i}}\right)=0, \\
\lambda_{2} \cdot H\left(\alpha_{i}, \beta_{i}, P_{R_{i}}\right)=0, \\
\lambda_{3} \cdot I\left(\alpha_{i}, \beta_{i}, P_{R_{i}}\right)=0, \\
\lambda_{4} \cdot J\left(\alpha_{i}, \beta_{i}, P_{R_{i}}\right)=0 .
\end{gathered}
$$

The conditions for non-negativity read $\alpha_{i}, \beta_{i}, P_{R_{i}}, \lambda_{1}, \lambda_{2}, \lambda_{3}, \lambda_{4} \geq 0$. It is straightforward to verify that if $\lambda_{3} \neq 0$, then $I\left(\alpha_{i}, \beta_{i}, P_{R_{i}}\right)=0$ implying that $\alpha_{i}=1$. Since this is not a feasible solution, therefore $\lambda_{3}=0$. Similarly, it can be shown that $\lambda_{4}=0$.

The final solution can be postulated in the following theorem.

Theorem 1: If $\lambda_{1} \neq 0 \Longrightarrow G\left(x, P_{R_{i}}\right)=0 ; \lambda_{2} \neq 0 \Longrightarrow$ $H\left(x, P_{R_{i}}\right)=0$, then we obtain the following optimal values

$$
\begin{aligned}
P_{R_{i}}=(\exp (\mathcal{W} & \left(\frac{\mathcal{A}}{B} \exp \left(-\frac{1}{B} \log ^{2}(2)\right) * \log (2)\right) \\
+ & \left.\left.\frac{\log ^{2}(2)}{B}\right)-1\right)\left(\frac{\sigma_{n_{D}}^{2}}{d_{R_{i}, D}^{-\vartheta}\left|h_{R_{i}, D}\right|^{2}}\right),
\end{aligned}
$$

where $\mathcal{A}=\frac{\left(\ln (2) d_{R_{i}, D}^{-\vartheta}\left|h_{R_{i}, D}\right|^{2}\right)\left(\zeta\left(P_{S} d_{S, R_{i}}^{-\vartheta}\left|h_{S, R_{i}}\right|^{2}+\sigma_{n_{R_{i}}}^{2}\right)\right)}{\sigma_{n_{D}}^{2}}$ and $\mathcal{W}(\cdot)$ is the Lambert $\mathrm{W}$ function $[3 \overline{3}]$.

$$
\beta_{i}=\frac{\varphi_{1} \cdot \varphi_{2}+P_{R_{i}}-E_{e x t} \cdot \varphi_{3}}{\varphi_{1} \cdot \varphi_{2}+\varphi_{2} \cdot \varphi_{3}+P_{R_{i}} \cdot \varphi_{3}}
$$

where $\varphi_{1}=B \log _{2}\left(1+\gamma_{R_{i}, D}\right), \varphi_{2}=\zeta\left(P_{S} d_{S, R_{i}}^{-\vartheta}\left|h_{S, R_{i}}\right|^{2}+\right.$ $\left.\sigma_{n_{R_{i}}}^{2}\right)$, and $\varphi_{3}=B \log _{2}\left(1+\gamma_{S, R_{i}}\right)+\left(\delta_{i} \cdot r\right)$.

$$
\alpha_{i}=\frac{\left(1-\beta_{i}\right) P_{R_{i}}-E_{e x t}}{\zeta \beta_{i}\left(P_{S} d_{S, R_{i}}^{-\vartheta}\left|h_{S, R_{i}}\right|^{2}+\sigma_{n_{R_{i}}}^{2}\right)} .
$$

Proof: See Appendix A.

Remark: Since there is one and only one feasible solution (as derived using the KKT conditions), therefore, the obtained solution must be in-line with respective conditions in Theorem 1. Alternatively if the conditions in Theorem 1 are not satisfied, then it is reasonable to discard the solution as the problem becomes infeasible.

It is proved in our analysis above (9) - 21) that the necessary conditions for KKT are satisfied. Assume that KKT yields the following solutions: (i) Primal variables: $\alpha_{i}^{\star}, \beta_{i}^{\star}, P_{R_{i}}^{\star}$, (ii) Dual Variables: $\lambda_{1}^{\star}, \lambda_{2}^{\star}$. The second order derivatives can therefore be represented using these solutions as: $\nabla_{\alpha_{i} \alpha_{i}}^{2} \mathcal{L}\left(\alpha_{i}^{\star}, \beta_{i}^{\star}, P_{R_{i}}^{\star} ; \lambda_{1}^{\star}, \lambda_{2}^{\star}\right)=$ $0, \quad \nabla_{\beta_{i} \beta_{i}}^{2} \mathcal{L}\left(\alpha_{i}^{\star}, \beta_{i}^{\star}, P_{R_{i}}^{\star} ; \lambda_{1}^{\star}, \lambda_{2}^{\star}\right)=0, \quad=\quad$ and $\nabla_{P_{R_{i}} P_{R_{i}}}^{2} \mathcal{L}\left(\alpha_{i}^{\star}, \beta_{i}^{\star}, P_{R_{i}}^{\star} ; \lambda_{1}^{\star}, \lambda_{2}^{\star}\right) \geq 0$. It is found that the sufficiency criteria are not strictly met and hence the function is not strictly constrained local minimum. However, the sufficiency conditions are satisfied, the solutions obtained above does not guarantee global optimality.

From an economic view-point, the Lagrange Multipliers $\lambda_{1}$ and $\lambda_{2}$ can be expounded as the prices for data and energy in cost/bit and cost/Joule, respectively. Leveraging the results from our analysis in Appendix A, we find that $\lambda_{1}=$ $\frac{f_{1}\left(P_{S}\right)}{f_{2}\left(P_{S}, \delta_{i}, r\right)} \cdot \lambda_{2}$, where $f_{1}\left(P_{S}\right)=\zeta\left(P_{S} d_{S, R_{i}}^{-\vartheta}\left|h_{S, R_{i}}\right|^{2}+\sigma_{n_{R_{i}}}^{2}\right)$, and $f_{2}\left(P_{S}, \delta_{i}, r\right)=B \log _{2}\left(1+\gamma_{S, R_{i}}\right)+\left(\delta_{i} \cdot r\right)$. Correspondingly, it is clear that if more data rate is demanded by the user provided the caching capacity is fixed, then we are enforced to compensate for the request by using the energy metric per cost unit in order to satisfy the respective data and energy constraints in $(P 2)$. This action would however add more to the energy price. Similarly, it is apparent that increasing the transmit power will readily add to the cost of data transfer in addition to an increased energy price. In the context of caching, it would be needless to mention that the higher the cache capacity is, the lower will be the prices for data and energy transmissions.

\section{B. Relay Selection}

In this subsection, we consider optimal selection of a relay to address the solution of outer optimization of $(P 1)$. Based on the above developments, we find the best relay which provides maximum throughput corresponding to (7). The best relay index is selected as $j^{\star}=\arg \max _{j \in\{1, \ldots, K\}}\left(1-\beta_{j}^{\star}\right) \hat{R}_{R_{j}, D}^{\star}$, where $\beta_{j}^{\star}$ and $\hat{R}_{R_{j}, D}^{\star}$ are the solutions of problem 8 . It is worth to mention that this relay selection is based on exhausted search and provides the best performance with high cost of complexity. Finding a compromise relay selection is of interest in the future research.

\section{Maximization of the Energy Stored at the RELAY}

In this section, we aim at maximizing the energy stored at the relay. The stored energy is calculated by subtracting the input energy, e.g., $E_{\text {ext }}$ plus the harvested energy, by the output energy, e.g., used for forwarding information to the destination. Our motivation behind this section is that the stored energy at the relay can be used to perform extra processing task, e.g., sensing, or to recharge a battery for future use. In particular, an optimization problem is formulated to jointly select the best relay and maximize the stored energy, while satisfying a given QoS.

For convenience, we introduce a new convention for fractions of time in the TS scheme, as shown in Fig. 4. However, the corresponding inter-relationships between the two conventions in Fig. 3 and Fig. 4 are established in Appendix C] For the newly adopted convention, the link between the transmitter 


\begin{tabular}{|c|c|c|}
\hline$\theta_{i} T$ & $\phi_{i} T$ & $\left(1-\left(\theta_{i}+\phi_{i}\right)\right) T$ \\
\hline $\begin{array}{l}\text { Energy Transmission } \\
\text { from Source to } i \text {-th Relay }\end{array}$ & $\begin{array}{l}\text { Information Transmission } \\
\text { from Source to } i \text {-th Relay, } \\
\text { Caching, and Re-encoding }\end{array}$ & $\begin{array}{l}\text { Information Transmission } \\
\text { from } i \text {-th Relay to the Destination }\end{array}$ \\
\hline
\end{tabular}

Fig. 4: Convention assumed for distribution of time to investigate the stored energy maximization problem.

and relay with cache is considered to be active for a fraction of $\left(\theta_{i}+\phi_{i}\right) T$ seconds, while the link between the relay and destination is active for the remaining $\left(1-\left(\theta_{i}+\phi_{i}\right)\right) T$, where $0 \leq \theta_{i}+\phi_{i} \leq 1$. As mentioned earlier, since the relay adopts a TS type of scheme for SWIPT, we assume that the energy harvesting at the relay takes place for a fraction of $\theta_{i} T$ seconds and the information decoding at the relay takes place for a fraction of $\phi_{i} T$ seconds. Similarly, we assume normalized time to use energy and power interchangeably without loss in generality. We remind that the harvested energy at the relay $i$ is given as

$$
E_{R_{i}}=\zeta \theta_{i}\left(P_{S} d_{S, R_{i}}^{-\vartheta}\left|h_{S, R_{i}}\right|^{2}+\sigma_{n_{R_{i}}}^{2}\right),
$$

where $\zeta$ is the energy conversion efficiency of the receiver.

We now consider the problem of relay selection for maximization of the energy stored at the relay, while ensuring that the requested rate between relay-destination is above a given threshold and that the total transmit powers at the transmitter and relay does not exceed a given limit. The corresponding optimization problem (P3) can be expressed as

$$
\begin{aligned}
&(P 3): \max _{i \in \mathcal{K}, \theta_{i}, \phi_{i}, P_{R_{i}}} {\left[\zeta \theta_{i}\left(P_{S} d_{S, R_{i}}^{-\vartheta}\left|h_{S, R_{i}}\right|^{2}+\sigma_{n_{R_{i}}}^{2}\right)\right.} \\
&\left.+E_{\text {ext }}-\left(1-\left(\theta_{i}+\phi_{i}\right)\right) P_{R_{i}}\right]^{+} \\
& \text {subject to : } \quad(C 1): \phi_{i}\left(\hat{R}_{S, R_{i}}+\left(\delta_{i} \cdot r\right)\right) \geq\left(1-\left(\theta_{i}+\phi_{i}\right)\right) \hat{R}_{R_{i}, D} \\
&(C 2):\left(1-\left(\theta_{i}+\phi_{i}\right)\right) P_{R_{i}} \leq E_{R_{i}} \\
&+E_{\text {ext }}, \\
&(C 3):\left(1-\left(\theta_{i}+\phi_{i}\right)\right) \hat{R}_{R_{i}, D} \geq r \\
&(C 4): 0<P_{S} \leq P^{\star} \\
&(C 5): 0 \leq \theta_{i}+\phi_{i} \leq 1
\end{aligned}
$$

where the objective in 25 is non-zero and the constraint 25c is to satisfy the QoS requirement.

The problem $(P 3)$ is difficult to solve, since it is a mixedinteger programming problem involving relay selection along with joint computations of $\theta_{i}, \phi_{i}$, and $P_{R_{i}}$. So, we recast $(P 3)$ into pair of coupled optimization problems for performing the outer optimization to choose the best relay, and inner optimization for joint computations of $\theta_{i}, \phi_{i}$, and $P_{R_{i}}$. In the following subsections, we address the optimal solutions to the inner and outer optimizations, respectively.

\section{A. Optimization of TS Factors and the Relay Transmit Power}

In this subsection, we consider the inner optimization problem of (P3). We determine the technique for joint computations of $\theta_{i}, \phi_{i}$, and $P_{R_{i}}$, for maximizing the energy stored at the relay while ensuring that the requested rate between relaydestination is above a given threshold and that the total transmit powers at the transmitter and relay does not exceed a given limit. Correspondingly, the sub-problem $(P 4)$ can be formulated as

$$
\begin{aligned}
(P 4): \max _{\theta_{i}, \phi_{i}, P_{R_{i}}} & {\left[\zeta \theta_{i}\left(P_{S} d_{S, R_{i}}^{-\vartheta}\left|h_{S, R_{i}}\right|^{2}+\sigma_{n_{R_{i}}}^{2}\right)+E_{\text {ext }}\right.} \\
& \left.-\left(1-\left(\theta_{i}+\phi_{i}\right)\right) P_{R_{i}}\right]^{+} \\
\text {subject to : } & (C 1): \phi_{i}\left(\hat{R}_{S, R_{i}}+\left(\delta_{i} \cdot r\right)\right) \\
& \geq\left(1-\left(\theta_{i}+\phi_{i}\right)\right) \hat{R}_{R_{i}, D}, \\
& (C 2):\left(1-\left(\theta_{i}+\phi_{i}\right)\right) P_{R_{i}} \leq E_{R_{i}}+E_{\text {ext }}, \\
& (C 3):\left(1-\left(\theta_{i}+\phi_{i}\right)\right) \hat{R}_{R_{i}, D} \geq r, \\
& (C 4): 0<P_{S} \leq P^{\star}, \\
& (C 5): 0 \leq \theta_{i}+\phi_{i} \leq 1 .
\end{aligned}
$$

This is a non-linear programming problem involving joint computations of $\theta_{i}, \phi_{i}$, and $P_{R_{i}}$, which introduces intractability. Therefore, we propose to solve this problem using the Karush-Kuhn-Tucker (KKT) conditions.

The Lagrangian for $(P 4)$ can be expressed as follows

$$
\begin{aligned}
& \mathcal{L}\left(\theta_{i}, \phi_{i}, P_{R_{i}} ; \mu_{1}, \mu_{2}, \mu_{3}, \mu_{4}\right)=F\left(\theta_{i}, \phi_{i}, P_{R_{i}}\right) \\
& -\mu_{1} G\left(\theta_{i}, \phi_{i}, P_{R_{i}}\right)-\mu_{2} H\left(\theta_{i}, \phi_{i}, P_{R_{i}}\right) \\
& -\mu_{3} I\left(\theta_{i}, \phi_{i}, P_{R_{i}}\right)-\mu_{4} J\left(\theta_{i}, \phi_{i}, P_{R_{i}}\right),
\end{aligned}
$$

where

$$
\begin{aligned}
F\left(\theta_{i}, \phi_{i}, P_{R_{i}}\right)= & \zeta \theta_{i}\left(P_{S} d_{S, R_{i}}^{-\vartheta}\left|h_{S, R_{i}}\right|^{2}+\sigma_{n_{R_{i}}}^{2}\right) \\
& +E_{\text {ext }}-\left(1-\left(\theta_{i}+\phi_{i}\right)\right) P_{R_{i}}, \\
G\left(\theta_{i}, \phi_{i}, P_{R_{i}}\right)= & \left(1-\left(\theta_{i}+\phi_{i}\right)\right) B \log _{2}\left(1+\gamma_{R_{i}, D}\right) \\
& -\phi_{i}\left[B \log _{2}\left(1+\gamma_{S, R_{i}}\right)+\left(\delta_{i} \cdot r\right)\right] \leq 0 \\
H\left(\theta_{i}, \phi_{i}, P_{R_{i}}\right)= & \left(1-\left(\theta_{i}+\phi_{i}\right)\right) P_{R_{i}}-E_{\text {ext }} \\
& -\zeta \theta_{i}\left(P_{S} d_{S, R_{i}}^{-\vartheta}\left|h_{S, R_{i}}\right|^{2}+\sigma_{n_{R_{i}}}^{2}\right) \leq 0, \\
I\left(\theta_{i}, \phi_{i}, P_{R_{i}}\right)= & r-\left(1-\left(\theta_{i}+\phi_{i}\right)\right) B \log _{2}\left(1+\gamma_{R_{i}, D}\right) \leq 0(31) \\
J\left(\theta_{i}, \phi_{i}, P_{R_{i}}\right)= & \left(\theta_{i}+\phi_{i}\right)-1 \leq 0 .
\end{aligned}
$$

with $\mu_{1}, \mu_{2}, \mu_{3}, \mu_{4}$ being the Lagrange Multipliers for the corresponding constraints $(\mathrm{C} 1),(\mathrm{C} 2),(\mathrm{C} 3)$, and (C5). Note that the objective function in 26 is non-differentiable, and hence we relax the $(x)^{+}=\max (0, x)$ constraint as represented in 28. However, only positive values should be the acceptable solutions as the problem becomes infeasible with negative values.

For optimality, $\nabla \mathcal{L}\left(\theta_{i}, \phi_{i}, P_{R_{i}} ; \mu_{1}, \mu_{2}, \mu_{3}, \mu_{4}\right)=0$. Thus, we can represent the equations for satisfying the optimality conditions as

$$
\begin{aligned}
& \frac{\partial \mathcal{L}\left(\theta_{i}, \phi_{i}, P_{R_{i}} ; \mu_{1}, \mu_{2}, \mu_{3}, \mu_{4}\right)}{\partial \theta_{i}} \Longrightarrow\left[\zeta \left(P_{S} d_{S, R_{i}}^{-\vartheta}\left|h_{S, R_{i}}\right|^{2}\right.\right. \\
& \left.\left.+\sigma_{n_{R_{i}}}^{2}\right)+P_{R_{i}}\right]-\mu_{1}\left[-B \log _{2}\left(1+\gamma_{R_{i}, D}\right)\right] \\
& -\mu_{2}\left[-P_{R_{i}}-\zeta\left(P_{S} d_{S, R_{i}}^{-\vartheta}\left|h_{S, R_{i}}\right|^{2}+\sigma_{n_{R_{i}}}^{2}\right)\right] \\
& -\mu_{3}\left[B \log _{2}\left(1+\gamma_{R_{i}, D}\right)\right]-\mu_{4}=0, \\
& \frac{\partial \mathcal{L}\left(\theta_{i}, \phi_{i}, P_{R_{i}} ; \mu_{1}, \mu_{2}, \mu_{3}, \mu_{4}\right)}{\partial \phi_{i}} \Longrightarrow P_{R_{i}}-\mu_{1}\left[-B \log _{2}(1+\right. \\
& \left.\left.\gamma_{R_{i}, D}\right)-\left(B \log _{2}\left(1+\gamma_{S, R_{i}}\right)+\left(\delta_{i} \cdot r\right)\right)\right] \\
& -\mu_{2}\left[-P_{R_{i}}\right]-\mu_{3}\left[B \log _{2}\left(1+\gamma_{R_{i}, D}\right)\right]-\mu_{4}=0 \text {, }
\end{aligned}
$$




$$
\begin{gathered}
\frac{\partial \mathcal{L}\left(\theta_{i}, \phi_{i}, P_{R_{i}} ; \mu_{1}, \mu_{2}, \mu_{3}, \mu_{4}\right)}{\partial P_{R_{i}}} \Longrightarrow-\left(1-\left(\theta_{i}+\phi_{i}\right)\right) \\
-\mu_{1}\left[\left(1-\left(\theta_{i}+\phi_{i}\right)\right)\left(\frac{\ln (2) d_{R_{i}, D}^{-\vartheta}\left|h_{R_{i}, D}\right|^{2}}{\sigma_{n_{D}}^{2}+P_{R_{i}} d_{R_{i}, D}^{-\vartheta}\left|h_{R_{i}, D}\right|^{2}}\right)\right] \\
-\mu_{2}\left(1-\left(\theta_{i}+\phi_{i}\right)\right)-\mu_{3}\left[-\left(1-\left(\theta_{i}+\phi_{i}\right)\right)\right. \\
\left.\left(\frac{\ln (2) d_{R_{i}, D}^{-\vartheta}\left|h_{R_{i}, D}\right|^{2}}{\sigma_{n_{D}}^{2}+P_{R_{i}} d_{R_{i}, D}^{-\vartheta}\left|h_{R_{i}, D}\right|^{2}}\right)\right]=0 .
\end{gathered}
$$

The conditions for feasibility are as expressed in (29), 30, (31), and (32). Complementary slackness expressions can be represented as follows

$$
\begin{gathered}
\mu_{1} \cdot G\left(\theta_{i}, \phi_{i}, P_{R_{i}}\right)=0 \\
\mu_{2} \cdot H\left(\theta_{i}, \phi_{i}, P_{R_{i}}\right)=0 \\
\mu_{3} \cdot I\left(\theta_{i}, \phi_{i}, P_{R_{i}}\right)=0 \\
\mu_{4} \cdot J\left(\theta_{i}, \phi_{i}, P_{R_{i}}\right)=0 .
\end{gathered}
$$

The conditions for non-negativity are: $\theta_{i}, \phi_{i}, P_{R_{i}}, \mu_{1}, \mu_{2}, \mu_{3}, \mu_{4} \geq 0$. It is clear that if $\mu_{4} \neq 0$, then $J\left(\theta_{i}, \phi_{i}, P_{R_{i}}\right)=0$ implying that $\theta_{i}+\phi_{i}=1$. Since this is not a feasible solution, therefore $\mu_{4}=0$. The two possible solution are as mentioned in the following theorems, respectively.

Theorem 2: If $\mu_{1} \neq 0 \Longrightarrow G\left(\theta_{i}, \phi_{i}, P_{R_{i}}\right)=0 ; \mu_{2}=$ $0 \Longrightarrow H\left(\theta_{i}, \phi_{i}, P_{R_{i}}\right) \neq 0 ; \mu_{3} \neq 0 \Longrightarrow I\left(\theta_{i}, \phi_{i}, P_{R_{i}}\right)=0$, then we obtain the following optimal values

$$
\begin{gathered}
P_{R_{i}}^{\dagger}=(\nu-1)\left(\frac{\sigma_{n_{D}}^{2}}{d_{R_{i}, D}^{-\vartheta}\left|h_{R_{i}, D}\right|^{2}}\right), \\
\phi_{i}^{\dagger}=\frac{r}{B \log _{2}\left(1+\gamma_{S, R_{i}}\right)+\left(\delta_{i} \cdot r\right)}, \\
\theta_{i}^{\dagger}=1-r\left(\frac{1}{B \log _{2}\left(1+\gamma_{S, R_{i}}\right)+\left(\delta_{i} \cdot r\right)}\right. \\
\left.+\frac{1}{B \log _{2}\left(1+\frac{P_{R_{i}}^{\dagger} d_{R_{i}, D}^{-\vartheta}\left|h_{R_{i}, D}\right|^{2}}{\sigma_{n_{D}}^{2}}\right)}\right)
\end{gathered}
$$

where

$$
\nu=\exp \left(\mathcal{W}\left(-\frac{\mathcal{A}}{B} \exp \left(-\frac{1}{B} \log ^{2}(2)\right) * \log (2)\right)+\frac{\log ^{2}(2)}{B}\right),
$$

with $\mathcal{A}=\ln (2)-\left(\frac{\zeta}{\sigma_{n_{D}}^{2}}\right)\left(\ln (2) d_{R_{i}, D}^{-\vartheta}\left|h_{R_{i}, D}\right|^{2}\right)\left(P_{S} d_{S, R_{i}}^{-\vartheta}\right.$ $\left.\left|h_{S, R_{i}}\right|^{2}+\sigma_{n_{R_{i}}}^{2}\right)$.

Theorem 3: If $\mu_{1} \neq 0 \Longrightarrow G\left(\theta_{i}, \phi_{i}, P_{R_{i}}\right)=0 ; \mu_{2} \neq$ $0 \Longrightarrow H\left(\theta_{i}, \phi_{i}, P_{R_{i}}\right)=0 ; \mu_{3} \neq 0 \Longrightarrow I\left(\theta_{i}, \phi_{i}, P_{R_{i}}\right)=0$, then the following values are optimal

$$
\begin{gathered}
P_{R_{i}}^{*}=\left(\eta_{L}-1\right)\left(\frac{\sigma_{n_{D}}^{2}}{d_{R_{i}, D}^{-\vartheta}\left|h_{R_{i}, D}\right|^{2}}\right), \\
\phi_{i}^{*}=\frac{r}{B \log _{2}\left(1+\gamma_{S, R_{i}}\right)+\left(\delta_{i} \cdot r\right)}, \\
\theta_{i}^{*}=\frac{r P_{R_{i}}^{*}-E_{e x t} B \log _{2}\left(1+\frac{P_{R_{i}}^{*} d_{R_{i}, D}^{-\vartheta}\left|h_{R_{i}, D}\right|^{2}}{\sigma_{n_{D}}^{2}}\right)}{\zeta\left(P_{S} d_{S, R_{i}}^{-\vartheta}\left|h_{S, R_{i}}\right|^{2}+\sigma_{n_{R_{i}}}^{2}\right)},
\end{gathered}
$$

where $\eta_{L}=$ Largest Root of $\left[\mathcal{A}-B \log _{2}(\eta)(\mathcal{B}+\mathcal{C} \eta+\right.$ $\left.\left.\mathcal{D} B \log _{2}(\eta)\right)=0\right]$, with $\mathcal{A}=a \cdot b \cdot r, \mathcal{B}=a \cdot b+a \cdot r$. $\left(\frac{\sigma_{n_{D}}^{2}}{d_{R_{i}, D}^{-\vartheta}\left|h_{R_{i}, D}\right|^{2}}\right)-b \cdot r, \mathcal{C}=-a \cdot r \cdot\left(\frac{\sigma_{n_{D}}^{2}}{d_{R_{i}, D}^{-\vartheta}\left|h_{R_{i}, D}\right|^{2}}\right)$, and $\mathcal{D}=a \cdot E_{\text {ext }}$, where $a=B \log _{2}\left(1+\gamma_{S, R_{i}}\right)+\left(\delta_{i} \cdot r\right)$, and $b=\zeta\left(P_{S} d_{S, R_{i}}^{-\vartheta}\left|h_{S, R_{i}}\right|^{2}+\sigma_{n_{R_{i}}}^{2}\right)$.

Proof: See Appendix B.

To summarize the solutions obtained above, we propose the following algorithm to maximize the stored energy in the relay supporting SWIPT - Caching system (MSE-WC Algorithm)

\section{Algorithm. MSE-WC Algorithm}

Input: The parameters $h_{S, R_{i}}, h_{R_{i}, D}, \delta_{i}, r$, and $E_{\text {ext }}$.

Output: The maximized value of energy stored at the relay: $\left\{E_{S}\right\}$.

1) : Initialize: $\zeta \in(0,1], P_{T} \in\left(0, \varepsilon P_{\text {Max }}\right], 0.5<\varepsilon<1$, $\sigma_{n_{R_{i}}}^{2}=1$, and $\sigma_{n_{D}}^{2}=1$.

2) : Compute $P_{R_{i}}^{\dagger}, \phi_{i}^{\dagger}$, and $\theta_{i}^{\dagger}$ using 40, 41, and (42) respectively.

$3)$ : Define: $E_{S}^{\dagger}=\zeta \theta_{i}^{\dagger}\left(P_{S} d_{S, R_{i}}^{-\vartheta}\left|h_{S, R_{i}}\right|^{2}+\sigma_{n_{R_{i}}}^{2}\right)+E_{\text {ext }}-(1-$ $\left.\left(\theta_{i}^{\dagger}+\phi_{i}^{\dagger}\right)\right) P_{R_{i}}^{\dagger}$.

4) : Compute $P_{R_{i}}^{*^{i}}, \phi_{i}^{*}$, and $\theta_{i}^{*}$ using 44, 45, and 46 respectively.

5) : Define: $E_{S}^{*}=\zeta \theta_{i}^{*}\left(P_{S} d_{S, R_{i}}^{-\vartheta}\left|h_{S, R_{i}}\right|^{2}+\sigma_{n_{R_{i}}}^{2}\right)+E_{\text {ext }}-(1-$ $\left.\left(\theta_{i}^{*}+\phi_{i}^{*}\right)\right) P_{R_{i}}^{*}$.

6) $: E_{S}=\max \left(E_{S}^{\dagger}, E_{S}^{*}\right)$.

7) : return $E_{S}$.

The algorithm proposed above returns the maximized value of the objective function as its output. First, we initialize all the necessary values as indicated in 1). Then, we compute the optimal values of $P_{R_{i}}^{\dagger}, \phi_{i}^{\dagger}$, and $\theta_{i}^{\dagger}$ in 2), and define the energy stored at the relay in 3). 2) and 3) corresponds to the solutions obtained for Case VI during the analysis. Similarly, we find the optimal values of $P_{R_{i}}^{*}, \phi_{i}^{*}$, and $\theta_{i}^{*}$ in 4), and define the energy stored at the relay in 5) accordingly. 4) and 5) corresponds to the solutions obtained for Case VIII during the analysis. Next, we find the maximum of the two computed local optimal solutions for the energy stored at the relay, which in turn maximizes the objective function. It should also be noted that the solutions proposed in (P2) for maximizing the energy stored at the relay are not necessarily global optimum, as the problem is non-linear in nature. However, the KKT conditions guarantees the local optimal solutions.

From our analysis above (27)-39), it is clear that the necessary conditions for KKT are satisfied. Let us assume that the optimal solutions obtained via KKT are (i) Primal variables: $\theta_{i}^{\star}, \phi_{i}^{\star}, P_{R_{i}}^{\star}$, (ii) Dual Variables: $\mu_{1}^{\star}, \mu_{2}^{\star}, \mu_{3}^{\star}$. With the help of these solutions, we find that the second order derivatives : $\nabla_{\theta_{i} \theta_{i}}^{2} \mathcal{L}\left(\theta_{i}^{\star}, \phi_{i}^{\star}, P_{R_{i}}^{\star} ; \mu_{1}^{\star}, \mu_{2}^{\star}, \mu_{3}^{\star}\right)=$ $0, \quad \nabla_{\phi_{i} \phi_{i}}^{2} \mathcal{L}\left(\theta_{i}^{\star}, \phi_{i}^{\star}, P_{R_{i}}^{\star} ; \mu_{1}^{\star}, \mu_{2}^{\star}, \mu_{3}^{\star}\right)=0$, and $\nabla_{P_{R_{i}} P_{R_{i}}}^{2} \mathcal{L}\left(\theta_{i}^{\star}, \phi_{i}^{\star}, P_{R_{i}}^{\star} ; \mu_{1}^{\star}, \mu_{2}^{\star}, \mu_{3}^{\star}\right) \geq 0 ;$ are sufficient conditions for KKT, but does not meet the sufficiency criteria strictly. Hence, the function is not strictly constrained local minimum. Therefore, the presented solutions are not necessarily global optimal.

In order to analyze the proposed approach from an economic perspective, we denote the equivalent relationship between the Lagrange Multipliers $\mu_{1}$ and $\mu_{3}$ as $\mu_{R}$ and rename $\mu_{2}$ as $\mu_{E}$ which corresponds to the the prices for data and 


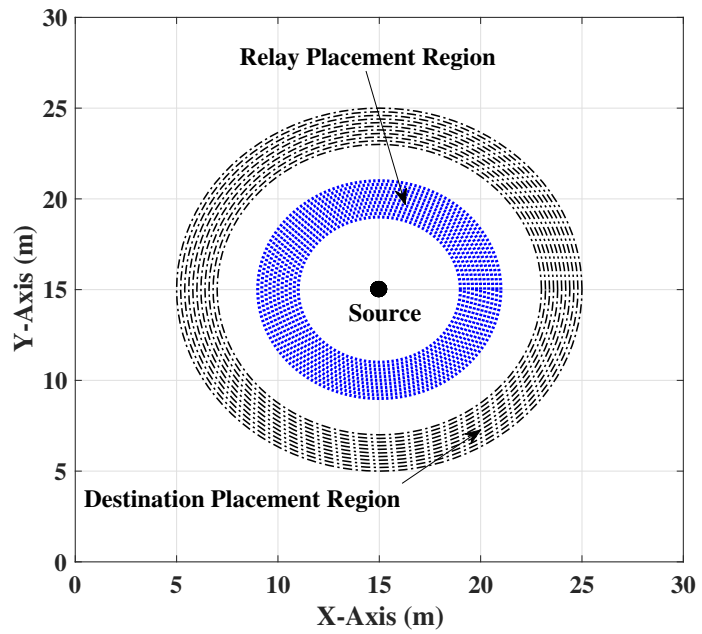

Fig. 5: Simulated Scenario: An ITU-R P.1238 framework implemented with the relays spatially distributed within the blue region $(4 \mathrm{~m}$ to $6 \mathrm{~m}$ from the source) and the destination placed randomly within the black region $(8 \mathrm{~m}$ to $10 \mathrm{~m}$ from the source).

energy, respectively, in cost units. Using the results from our analysis in Appendix B, we find that $\mu_{E}=\frac{f_{1}\left(P_{S}, \delta_{i}, r\right)}{f_{2}\left(P_{S}\right)} \cdot \mu_{R}$, where $f_{1}\left(P_{S}, \delta_{i}, r\right)$, and $f_{2}\left(P_{S}\right)$ are functions computed as per the illustrated technique. Correspondingly, if more energy is required by the relay with fixed caching capacity, then we are forced to compensate for the request by using the energy metric per cost unit at the source in order to satisfy the respective data and energy constraints in $(P 2)$. This action would however add more to the data price as well. Similarly, it is apparent that increasing the transmit power will readily add to the cost of energy transfer in addition to an increased data price. Furthermore in the context of caching, it is worth mentioning that extra cache capacity implies subordinate prices for data and energy transmissions per cost unit.

\section{B. Relay Selection}

From the methods proposed above, optimal TS ratios and the relay transmit power can be computed easily. Herein, we propose to find the best relay which provides maximized harvested power corresponding to 25]. In this context, the index of the optimally selected relay can be expressed as $j^{\star}=\arg \max _{j \in\{1, \ldots, K\}} E_{S_{j}}^{\star}$, where $E_{S_{j}}^{\star}$ is the optimal energy stored at the $j$-th relay as the solution of problem (26).

\section{Numerical Results}

In this section, we evaluate the performance of the proposed system for the solutions presented in this paper. We employ the ITU Radiocommunication Sector (ITU-R) P.1238 channel model with central frequency assumption at $450 \mathrm{MHz}$. The signal fading in both the hops follow Ricean distribution with $\mathrm{K}$-factor of 3.5. The overall emulation setup is depicted in Fig. 5. Additionally, we consider a total bandwidth of $B=1$ $\mathrm{MHz}, \zeta=0.50$ [34], $\sigma_{n_{R_{i}}}^{2}=-100 \mathrm{dBW}$, and $\sigma_{n_{D}}^{2}=-100$ $\mathrm{dBW}$. All the relays are assumed to have the same caching coefficient, i.e., $\delta_{i}=\delta, \forall i$. All the results are evaluated over 500 Monte-Carlo random channel realizations. The proposed architecture is compared with a reference scheme using a fix time-splitting. The reference scheme spends the first half

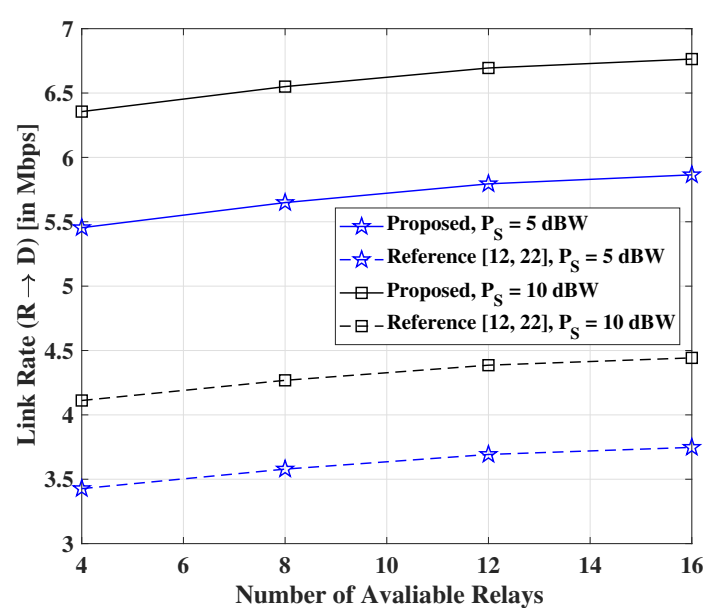

(a)

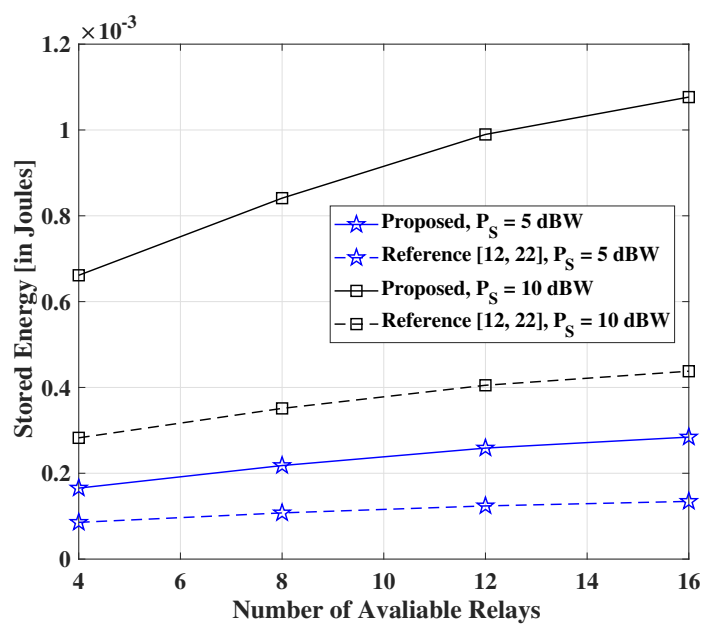

(b)

Fig. 6: Performance comparison between the proposed cache-aided SWIPT and the reference scheme for different number of available relays with $\delta=$ $0.5, r=3 \mathrm{Mbps}$, and $E_{\text {ext }}=1 \mu \mathrm{J}$. (a) Link rate performance. (b) Stored energy performance.

period for information broadcasting and energy harvesting, and uses the second half period for relaying [17], [30].

Fig. 6 plots the performance of the cache-aided SWIPT as a function of the total number of relays. The result is calculated based on the best relay selected as in Section IIII and Section IV] In both cases, $\delta=0.5$, and $E_{\text {ext }}=1 \mu \mathrm{J}$. It is observed from Fig. 6(a) that the proposed architecture significantly outperforms the reference and the gain is larger as the number of available relays increases. In particular, the proposed architecture achieves a performance gain of $15 \%$ for $P_{S}=5 \mathrm{dBW}$ and $20 \%$ for $P_{S}=10 \mathrm{dBW}$ over the reference scheme. This result confirms the effectiveness of the proposed optimization framework. It is also shown that having more relays results in a better serving rate thanks to inherent diversity gain brought by the relays. The harvested energy comparison between the proposed and the reference is plotted in Fig. 6(b). A similar conclusion is observed that the proposed architecture surpasses the reference in all cases. In addition, having more available relays improves the harvested energy.

Fig. 77a) illustrates the results corresponding to the solutions proposed for the rate maximization problem, assuming that an 


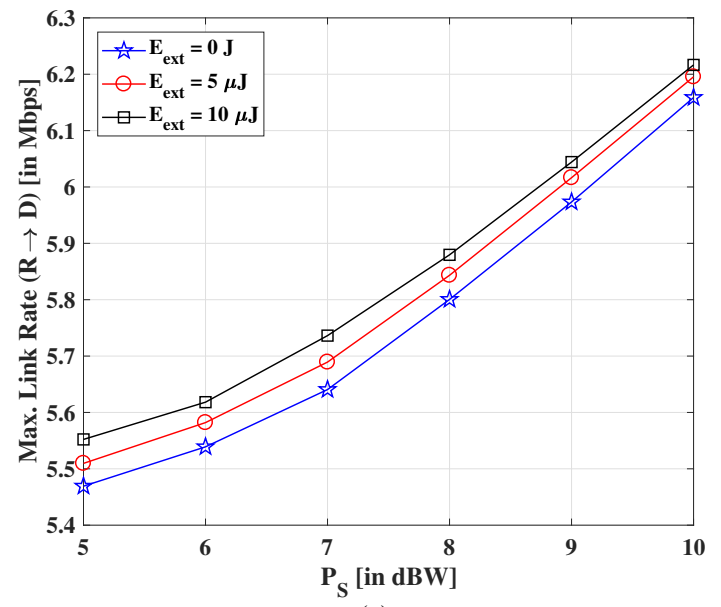

(a)

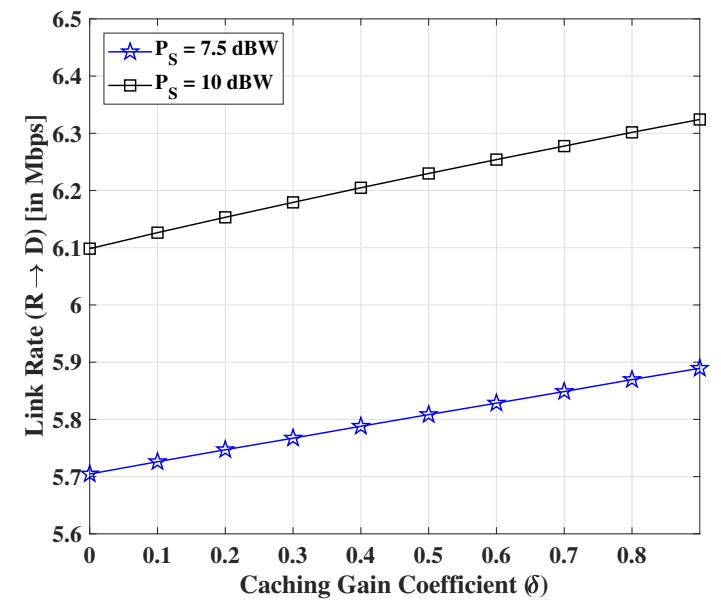

(b)

Fig. 7: Link-rate maximization: (a) Maximum link rate versus increasing values of $P_{S}$ for various values of $E_{\text {ext }}$ with total number of available relays $K=8$, caching coefficient $\delta=0.5$, and $r=5$ Mbps. (b) Maximized link rate versus the caching coefficient for various $P_{S}$ with total number of available relays $K=8, E_{\text {ext }}=10 \mu \mathrm{J}$, and $r=5$ Mbps.

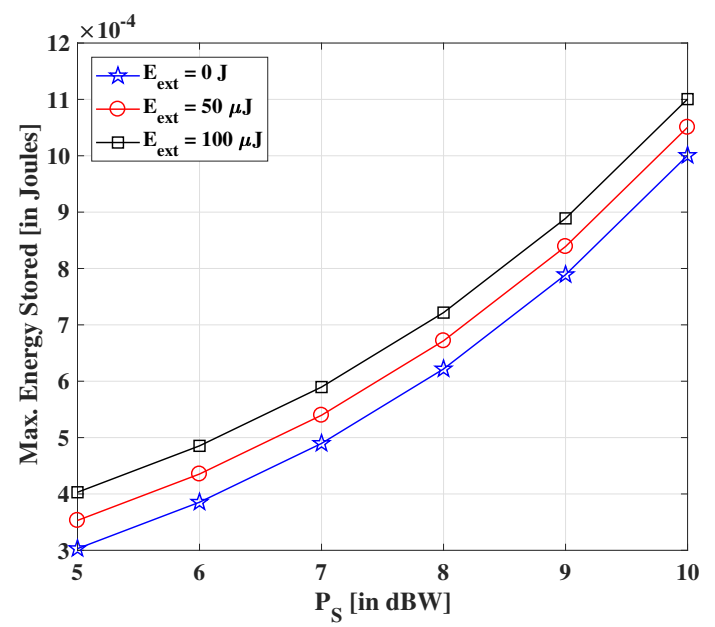

(a)

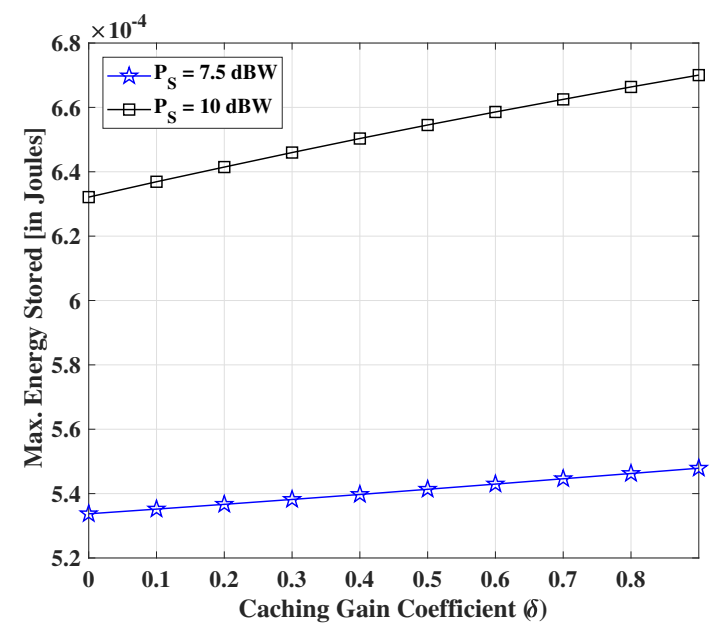

(b)

Fig. 8: Stored Energy maximization: (a) Stored energy performance as a function of the source transmit power for various values of $E_{\text {ext }}$. $K=8$ relays, $\delta=0.5$ and $r=1 \mathrm{Mbps}$. (b) Stored energy performance as a function of the caching coefficient for various values of $P_{S} . K=8$ relays, $E_{\text {ext }}=0.5$ mJ, and $r=5$ Mbps.

optimal relay is chosen as per the solutions corresponding to the outer optimization of (7). It is observed from the figure that the source transmit power has a significant influence on the achievable rate. In particular, by increasing the source transmit power by $5 \mathrm{dBW}$, the serving rate is increased by $30 \%$. This result can be explained from the fact that for a given caching coefficient, the source does not have to send the whole requested content to the relay. In this case, having a larger source power results in more harvested energy, which in turn increase the relay's transmit power. Especially, this observation is also obtained when there is not external energy source, e.g., $E_{\text {ext }}=0$, which shows the effectiveness of the proposed cache-aided SWIPT architecture.

Fig. 7. (b) plots the maximum throughput as a function of the caching gain coefficient with $E_{\text {ext }}=10 \mu \mathrm{J}$ and $r=5 \mathrm{Mbps}$. It is observed that the caching gain has similar impact on the achievable throughput for different values of $P_{S}$. In general, a larger cache size (or equivalent larger caching coefficient) results in a higher serving rate. This result together with result in Fig. 77a) suggest an interactive role of the caching capacity and the transmit power. In particular, a smaller source power system can still achieve the same throughput by increasing the cache size.

Fig. 8(a) presents the stored energy at the chosen relay, according to the solution of outer optimization of 25], as a function of the source's transmit power and different external energy values. It is shown from the results that the source transmit power has large impacts on the stored energy at the relay. In particular, increasing the source's transmit power by $2 \mathrm{dBW}$ will double the stored energy at the relay. It is also observed that increasing the external energy can significantly improve the stored energy at high $P_{S}$ values. However, when $P_{S}$ is small, increasing $E_{\text {ext }}$ does not bring considerable improvement. This is because at low $P_{S}$ values, most of the time is used for information transfer from the source to the relay.

Fig. 8 (b) depicts the plot of the energy stored at the selected relay as a function of the cache capacity $\delta$, with $E_{\text {ext }}=0.5$ 


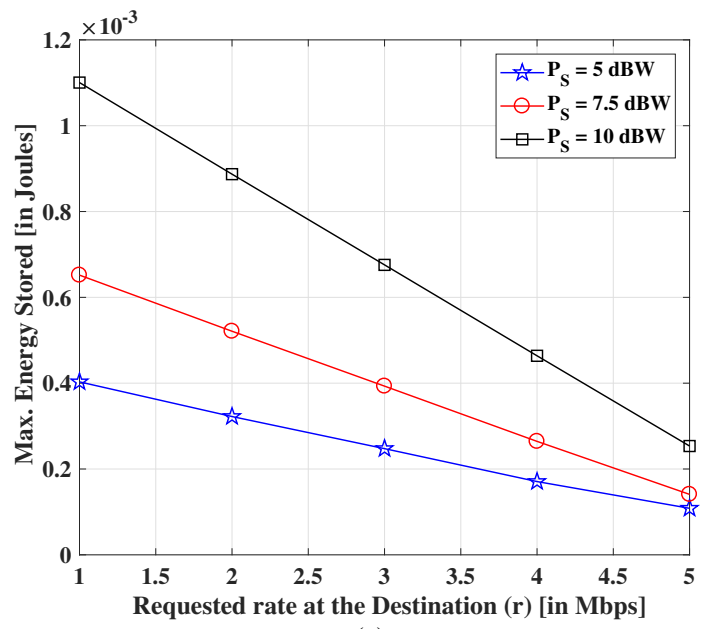

(a)

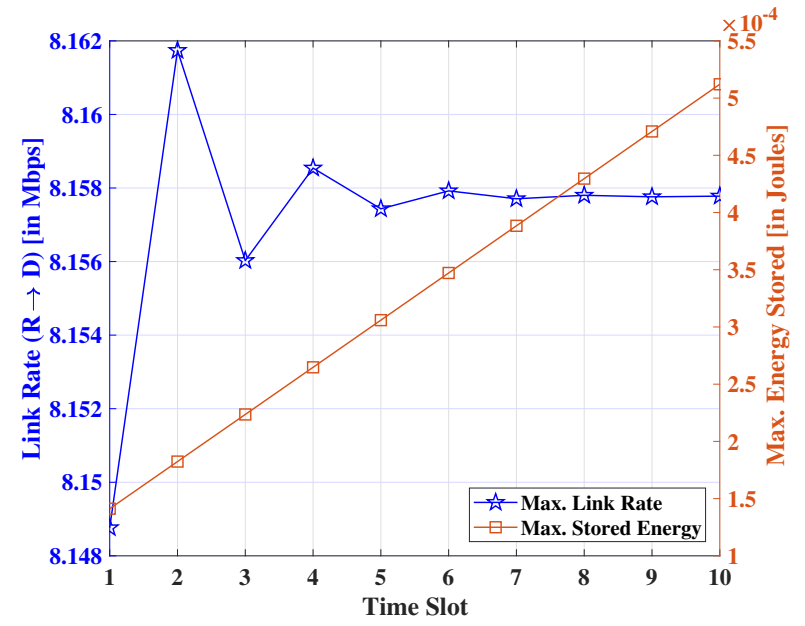

(b)

Fig. 9: Rate-Energy trade-off and comparison of the two proposed relay selection schemes. (a) Stored energy performance as a function of QoS requirements with different source transmit power. $K=8$ relays, $\delta=0.5$ and $E_{\text {ext }}=0.1 \mathrm{~mJ}$. (b) Performance of the two proposed relay selection schemes for ten time slots. $K=8$ relays, $P_{S}=7.5 \mathrm{dBW}, E_{\text {ext }}=1 \mu \mathrm{J}, r=3 \mathrm{Mbps}$.

$\mathrm{mJ}$ and $r=5$ Mbps. The case with $\delta=0$ implies that there is no caching at the relay. It is shown that caching helps to increase the saved energy at the relay for all $P_{S}$ values. And the increased stored energy are almost similar for different $P_{S}$. This is because of the linear model of the caching system.

Fig. 9(a) presents an evaluation of the energy stored at the chosen relay against the increasing values of $r$. It is seen that the energy stored at the relay decreases with increasing values of requested rate $(r)$, for $\delta=0.5$ and $E_{\text {ext }}=0.1 \mathrm{~mJ}$. On the other hand, it is clear that with increasing values of $P_{S}$, the energy stored at the relay increases non-linearly. The former variation is due to the fact that in order to meet the demand of requested rate at the destination, more energy would be required for resource allocation at the relay which utilizes the harvested energy.

Fig. 9 (b) illustrates the comparison between the two proposed relay selection schemes for various time slots with $K=8$ relays, $P_{S}=7.5 \mathrm{dBW}, E_{\text {ext }}=1 \mu \mathrm{J}, r=3$ Mbps. Herein, we assumed $E_{\text {ext }}=1 \mu \mathrm{J}$ as the initial external energy in the first slot, while in the subsequent slots, the value for $E_{\text {ext }}$ was assumed as the stored energy value in the previous slot. The results helps in simultaneously studying impact of the relay selection using the two problems, where it is seen that the throughput saturates after some time-slots implying that $E_{\text {ext }} \rightarrow 0$ while the stored energy increases linearly. These respective cases for throughput and stored energy maximization provides useful insight on the benefits of relay selection on the system performance.

\section{CONCLUSION}

In this paper, we proposed and investigated relay selection strategy in a novel cache-assisted SWIPT architecture with dynamic time switching (TS) in dual-hop half duplex system, where the relays employ the DF protocol. We addressed the problem of relay selection to maximize the data throughput between the relay and destination under constraint on minimum energy stored at the relay; and relay selection for maximizing the energy stored at the relay under constraints on minimum rate and harvested energy, guaranteeing a good performance in both the cases with regards to the QoS constraints. Besides, both the problems were formulated according to two separate yet distinct conventions over the time period. We presented the closed-form solutions for the proposed relay system to enable SWIPT with caching. With the help of simulations, we illustrated the results corresponding to the solutions obtained for the aforementioned problems with parameter variations. This work can be further extended to many fascinating directions like multiuser and multicarrier scenario, and relaying with full duplexing mode. Another promising research topic is to consider multiple antennas systems where an additional dimension is how to optimally select antennas or design beamforming vectors for information transmission or energy transfer. In addition, the energy queuing model and adoption of Markov Decision Process (MDP) to model the aforementioned problems can be another interesting direction to this work, wherein the same problems can be studied using stochastic methods with uncertain CSI. Therefore, by leveraging from the benefits of caching, we intend to improve system efficiency to enhance SWIPT performance. This can be realized by dynamic adjustment of the TS factors, which can significantly improve the throughput and energy storage capabilities at the relay.

\section{APPENDIX A}

\section{ANALYSIS OF DIFFERENT POSSIBILITIES FROM KKT CONDITIONS For Data MAXiMization PROBLEM}

We analyze all the cases corresponding to $95-(21)$ in order to obtain a feasible solution as follows

Case I: $\lambda_{1}=0 \Longrightarrow G\left(\alpha_{i}, \beta_{i}, P_{R_{i}}\right) \neq 0 ; \lambda_{2} \neq 0 \Longrightarrow$ $H\left(\alpha_{i}, \beta_{i}, P_{R_{i}}\right)=0$

From (15), we find that $\lambda_{2}=0$, which is contradictory. Hence, this case is not possible.

Case II: $\lambda_{1}=0 \Longrightarrow G\left(\alpha_{i}, \beta_{i}, P_{R_{i}}\right) \neq 0 ; \lambda_{2}=0 \Longrightarrow$ $H\left(\alpha_{i}, \beta_{i}, P_{R_{i}}\right) \neq 0$

This case is not acceptable as $B \log _{2}\left(1+\gamma_{R_{i}, D}\right) \neq 0$.

Case III: $\lambda_{1} \neq 0 \Longrightarrow G\left(\alpha_{i}, \beta_{i}, P_{R_{i}}\right)=0 ; \lambda_{2}=0 \Longrightarrow$ $H\left(\alpha_{i}, \beta_{i}, P_{R_{i}}\right) \neq 0$ 
From (17), we find that $\lambda_{1}=1$ which implies that $P_{S} \rightarrow 0$. Therefore, this is not a feasible solution.

Case IV: $\lambda_{1} \neq 0 \Longrightarrow G\left(\alpha_{i}, \beta_{i}, P_{R_{i}}\right)=0 ; \lambda_{2} \neq 0 \Longrightarrow$ $H\left(\alpha_{i}, \beta_{i}, P_{R_{i}}\right)=0$

For $\lambda_{1} \neq 0 \Longrightarrow G\left(x, P_{R_{i}}\right)=0 ; \lambda_{2} \neq 0 \Longrightarrow H\left(x, P_{R_{i}}\right)=$ 0 , we deduce to the following equations

$$
\begin{gathered}
-\lambda_{1}\left[\beta_{i}\left(B \log _{2}\left(1+\gamma_{S, R_{i}}\right)+\left(\delta_{i} \cdot r\right)\right)\right]+\lambda_{2}\left[\zeta \beta _ { i } \left(P_{S} d_{S, R_{i}}^{-\vartheta}\left|h_{S, R_{i}}\right|^{2}\right.\right. \\
\left.\left.+\sigma_{n_{R_{i}}}^{2}\right)\right]=0, \quad(47) \\
-B \log _{2}\left(1+\gamma_{R_{i}, D}\right)+\lambda_{1}\left(B \log _{2}\left(1+\gamma_{R_{i}, D}\right)\right. \\
+\left(1-\alpha_{i}\right)\left(B \log _{2}\left(1+\gamma_{S, R_{i}}\right)+\left(\delta_{i} \cdot r\right)\right) \\
+\lambda_{2}\left(P_{R_{i}}+\zeta \alpha_{i}\left(P_{S} d_{S, R_{i}}^{-\vartheta}\left|h_{S, R_{i}}\right|^{2}+\sigma_{n_{R_{i}}}^{2}\right)\right)=0 \\
\frac{\ln (2) d_{R_{i}, D}^{-\vartheta}\left|h_{R_{i}, D}\right|^{2}}{\sigma_{n_{D}}^{2}+P_{R_{i}} d_{R_{i}, D}^{-\vartheta}\left|h_{R_{i}, D}\right|^{2}} \\
-\lambda_{1}\left(\frac{\ln (2) d_{R_{i}, D}^{-\vartheta}\left|h_{R_{i}, D}\right|^{2}}{\sigma_{n_{D}}^{2}+P_{R_{i}} d_{R_{i}, D}^{-\vartheta}\left|h_{R_{i}, D}\right|^{2}}\right)-\lambda_{2}=0 \\
\left(1-\beta_{i}\right) B \log _{2}\left(1+\gamma_{R_{i}, D}\right)-\left(1-\alpha_{i}\right) \beta_{i}\left(B \log _{2}\left(1+\gamma_{S, R_{i}}\right)\right. \\
\left.+\left(\delta_{i} \cdot r\right)\right)=0 . \\
\left(1-\beta_{i}\right) P_{R_{i}}-\zeta \alpha_{i} \beta_{i}\left(P_{S} d_{S, R_{i}}^{-\vartheta}\left|h_{S, R_{i}}\right|^{2}+\sigma_{n_{R_{i}}}^{2}\right)-E_{e x t}=0 .
\end{gathered}
$$

From 47) and 49, we have

$$
\begin{aligned}
\lambda_{1} & =\frac{\chi_{1}\left(\zeta\left(P_{S} d_{S, R_{i}}^{-\vartheta}\left|h_{S, R_{i}}\right|^{2}+\sigma_{n_{R_{i}}}^{2}\right)\right)}{\chi_{2}}, \\
\lambda_{2} & =\frac{\chi_{1}\left(B \log _{2}\left(1+\gamma_{S, R_{i}}\right)+\left(\delta_{i} \cdot r\right)\right)}{\chi_{2}},
\end{aligned}
$$

where $\chi_{1}=\left(\ln (2) d_{R_{i}, D}^{-\vartheta}\left|h_{R_{i}, D}\right|^{2}\right)$, and $\chi_{2}=$ $\left(\ln (2) d_{R_{i}, D}^{-\vartheta}\left|h_{R_{i}, D}\right|^{2}\right)\left(\zeta\left(P_{S} d_{S, R_{i}}^{-\vartheta}\left|h_{S, R_{i}}\right|^{2}+\sigma_{n_{R_{i}}}^{2}\right)\right)+\left(\sigma_{n_{D}}^{2}+\right.$ $\left.P_{R_{i}} d_{R_{i}, D}^{-\vartheta}\left|h_{R_{i}, D}\right|^{2}\right)\left(B \log _{2}\left(1+\gamma_{S, R_{i}}\right)+\left(\delta_{i} \cdot r\right)\right)$.

Assuming $\kappa=1+\gamma_{R_{i}, D}$ and substituting (52) and (53) in (48), we obtain the following equation

$$
\mathcal{A}+\kappa\left[\ln (2)-B \log _{2}(\kappa)\right]=0,
$$

where $\mathcal{A}=\frac{\left(\ln (2) d_{R_{i}, D}^{-\vartheta}\left|h_{R_{i}, D}\right|^{2}\right)\left(\zeta\left(P_{S} d_{S, R_{i}}^{-\vartheta}\left|h_{S, R_{i}}\right|^{2}+\sigma_{n_{R_{i}}}^{2}\right)\right)}{\sigma_{n_{D}}^{2}}$. The solution of this equation is obtained in a closed form as follows

$$
\kappa=\exp \left(\mathcal{W}\left(\frac{\mathcal{A}}{B} \exp \left(-\frac{1}{B} \log ^{2}(2)\right) * \log (2)\right)+\frac{\log ^{2}(2)}{B}\right),
$$

where $\mathcal{W}(\cdot)$ is the Lambert $\mathrm{W}$ function [33].

Using (55), 50) and (51), we obtain the following

$$
\beta_{i}=\frac{\varphi_{1} \cdot \varphi_{2}+P_{R_{i}}-E_{e x t} \cdot \varphi_{3}}{\varphi_{1} \cdot \varphi_{2}+\varphi_{2} \cdot \varphi_{3}+P_{R_{i}} \cdot \varphi_{3}}
$$

where $\varphi_{1}=B \log _{2}\left(1+\gamma_{R_{i}, D}\right), \varphi_{2}=\zeta\left(P_{S} d_{S, R_{i}}^{-\vartheta}\left|h_{S, R_{i}}\right|^{2}+\right.$ $\left.\sigma_{n_{R_{i}}}^{2}\right)$, and $\varphi_{3}=B \log _{2}\left(1+\gamma_{S, R_{i}}\right)+\left(\delta_{i} \cdot r\right)$.

$$
\alpha_{i}=\frac{\left(1-\beta_{i}\right) P_{R_{i}}-E_{e x t}}{\zeta \beta_{i}\left(P_{S} d_{S, R_{i}}^{-\vartheta}\left|h_{S, R_{i}}\right|^{2}+\sigma_{n_{R_{i}}}^{2}\right)} .
$$

\section{APPENDIX B}

\section{ANALYSIS OF DIFFERENT POSSIBILITIES FROM KKT}

CONDITIONS FOR MAXIMIZATION PROBlEM OF ENERGY STORED AT THE RELAY

We analyze all the cases in order to obtain a feasible solution corresponding to 27) - 39. The analysis is as follows

Case I: $\mu_{1}=0 \Longrightarrow G\left(\theta_{i}, \phi_{i}, P_{R_{i}}\right) \neq 0 ; \mu_{2}=0 \Longrightarrow$ $H\left(\theta_{i}, \phi_{i}, P_{R_{i}}\right) \neq 0 ; \mu_{3}=0 \Longrightarrow I\left(\theta_{i}, \phi_{i}, P_{R_{i}}\right) \neq 0$

From (33) and 234, we find that $P_{R_{i}}=-\zeta\left(P_{S}|g|^{2}+\sigma_{n_{R_{i}}}^{2}\right)$ or $P_{R_{i}}=0$ respectively. Since both these solutions cannot be accepted, therefore this case is not possible.

Case II: $\mu_{1}=0 \Longrightarrow G\left(\theta_{i}, \phi_{i}, P_{R_{i}}\right) \neq 0 ; \mu_{2}=0 \Longrightarrow$ $H\left(\theta_{i}, \phi_{i}, P_{R_{i}}\right) \neq 0 ; \mu_{3} \neq 0 \Longrightarrow I\left(\theta_{i}, \phi_{i}, P_{R_{i}}\right)=0$

This case again leads us to the unacceptable solution as in the previous case, therefore this case can be excluded.

Case III: $\mu_{1}=0 \Longrightarrow G\left(\theta_{i}, \phi_{i}, P_{R_{i}}\right) \neq 0 ; \mu_{2} \neq 0 \Longrightarrow$ $H\left(\theta_{i}, \phi_{i}, P_{R_{i}}\right)=0 ; \mu_{3}=0 \Longrightarrow I\left(\theta_{i}, \phi_{i}, P_{R_{i}}\right) \neq 0$

From the optimality conditions, we deduce that $\mu_{2}=1$ with no solutions for $\theta_{i}, \phi_{i}$, and $P_{R_{i}}$. Hence, this case is not admissible.

Case IV: $\mu_{1} \neq 0 \Longrightarrow G\left(\theta_{i}, \phi_{i}, P_{R_{i}}\right)=0 ; \mu_{2}=0 \Longrightarrow$ $H\left(\theta_{i}, \phi_{i}, P_{R_{i}}\right) \neq 0 ; \mu_{3}=0 \Longrightarrow I\left(\theta_{i}, \phi_{i}, P_{R_{i}}\right) \neq 0$

Herein, we find that $\mu_{1}<0$; which violates the non-negativity condition. Thus, this case is infeasible.

Case V: $\mu_{1}=0 \Longrightarrow G\left(\theta_{i}, \phi_{i}, P_{R_{i}}\right) \neq 0 ; \mu_{2} \neq 0 \Longrightarrow$ $H\left(\theta_{i}, \phi_{i}, P_{R_{i}}\right)=0 ; \mu_{3} \neq 0 \Longrightarrow I\left(\theta_{i}, \phi_{i}, P_{R_{i}}\right)=0$

For this case, we can represent the following equations in their simplified forms

$$
\begin{gathered}
{\left[\zeta\left(P_{S} d_{S, R_{i}}^{-\vartheta}\left|h_{S, R_{i}}\right|^{2}+\sigma_{n_{R_{i}}}^{2}\right)+P_{R_{i}}\right]+\mu_{2}\left[P_{R_{i}}\right.} \\
\left.+\zeta\left(P_{S} d_{S, R_{i}}^{-\vartheta}\left|h_{S, R_{i}}\right|^{2}+\sigma_{n_{R_{i}}}^{2}\right)\right]-\mu_{3}\left[B \log _{2}\left(1+\gamma_{R_{i}, D}\right)\right]=0 \\
P_{R_{i}}+\mu_{2}\left[P_{R_{i}}\right]-\mu_{3}\left[B \log _{2}\left(1+\gamma_{R_{i}, D}\right)\right]=0 \\
1+\mu_{2}-\mu_{3}\left(\frac{\ln (2) d_{R_{i}, D}^{-\vartheta}\left|h_{R_{i}, D}\right|^{2}}{\sigma_{n_{D}}^{2}+P_{R_{i}} d_{R_{i}, D}^{-\vartheta}\left|h_{R_{i}, D}\right|^{2}}\right)=0 \\
\left(1-\left(\theta_{i}+\phi_{i}\right)\right) P_{R_{i}}-\zeta \theta_{i}\left(P_{S} d_{S, R_{i}}^{-\vartheta}\left|h_{S, R_{i}}\right|^{2}+\sigma_{n_{R_{i}}}^{2}\right)-E_{e x t}=0, \\
r-\left(1-\left(\theta_{i}+\phi_{i}\right)\right) B \log _{2}\left(1+\gamma_{R_{i}, D}\right)=0 .
\end{gathered}
$$

Solving the above equations, we obtain solutions for $P_{R_{i}}$ as follows

$$
P_{R_{i}}=\max \left(\exp \left(\mathcal{W}\left(\exp \left(-\log ^{2}(2)\right)+\log (2)\right)+\log ^{2}(2)\right),\right.
$$$$
\left.\exp \left(\mathcal{W}_{-1}\left(\exp \left(-\log ^{2}(2)\right)+\log (2)\right)+\log ^{2}(2)\right)\right),
$$

where $\mathcal{W}(\cdot)$ is the LambertW function or the product log function and $\mathcal{W}_{k}(\cdot)$ is the analytic continuation of the product $\log$ function [33]. As the solution is independent of $P_{S}$, it is reasonable to discard this solution.

Case VI: $\mu_{1} \neq 0 \Longrightarrow G\left(\theta_{i}, \phi_{i}, P_{R_{i}}\right)=0 ; \mu_{2}=0 \Longrightarrow$ $H\left(\theta_{i}, \phi_{i}, P_{R_{i}}\right) \neq 0 ; \mu_{3} \neq 0 \Longrightarrow I\left(\theta_{i}, \phi_{i}, P_{R_{i}}\right)=0$

We can represent the following equations in their simplified forms

$$
\begin{aligned}
& {\left[\zeta\left(P_{S} d_{S, R_{i}}^{-\vartheta}\left|h_{S, R_{i}}\right|^{2}+\sigma_{n_{R_{i}}}^{2}\right)+P_{R_{i}}\right]} \\
& \quad+\mu_{1}\left[B \log _{2}\left(1+\gamma_{R_{i}, D}\right)\right]-\mu_{3}\left[B \log _{2}\left(1+\gamma_{R_{i}, D}\right)\right]=0
\end{aligned}
$$

$P_{R_{i}}+\mu_{1}\left[B \log _{2}\left(1+\gamma_{R_{i}, D}\right)+\left(B \log _{2}\left(1+\gamma_{S, R_{i}}\right)+\left(\delta_{i} \cdot r\right)\right)\right]$

$$
-\mu_{3}\left[B \log _{2}\left(1+\gamma_{R_{i}, D}\right)\right]=0 \text {, }
$$




$$
1+\mu_{2}-\mu_{3}\left(\frac{\ln (2) d_{R_{i}, D}^{-\vartheta}\left|h_{R_{i}, D}\right|^{2}}{\sigma_{n_{D}}^{2}+P_{R_{i}} d_{R_{i}, D}^{-\vartheta}\left|h_{R_{i}, D}\right|^{2}}\right)=0,
$$

$\left(1-\left(\theta_{i}+\phi_{i}\right)\right) P_{R_{i}}-\zeta \theta_{i}\left(P_{S} d_{S, R_{i}}^{-\vartheta}\left|h_{S, R_{i}}\right|^{2}+\sigma_{n_{R_{i}}}^{2}\right)-E_{e x t}=0$,

$$
r-\left(1-\left(\theta_{i}+\phi_{i}\right)\right) B \log _{2}\left(1+\gamma_{R_{i}, D}\right)=0 .
$$

From 64) and 65, we obtain

$$
\mu_{1}=\frac{\zeta\left(P_{S} d_{S, R_{i}}^{-\vartheta}\left|h_{S, R_{i}}\right|^{2}+\sigma_{n_{R_{i}}}^{2}\right)}{B \log _{2}\left(1+\gamma_{S, R_{i}}\right)+\left(\delta_{i} \cdot r\right)} .
$$

Substituting (69) in (66), we find the following

$$
\begin{aligned}
\mu_{3}=\left(\frac{\sigma_{n_{D}}^{2}+P_{R_{i}} d_{R_{i}, D}\left|h_{R_{i}, D}\right|^{2}}{\ln (2) d_{R_{i}, D}^{-\vartheta}\left|h_{R_{i}, D}\right|^{2}}\right) & \\
& +\left(\frac{\zeta\left(P_{S} d_{S, R_{i}}^{-\vartheta}\left|h_{S, R_{i}}\right|^{2}+\sigma_{n_{R_{i}}}^{2}\right)}{B \log _{2}\left(1+\gamma_{S, R_{i}}\right)+\left(\delta_{i} \cdot r\right)}\right) .
\end{aligned}
$$

Substituting (69) and (70) in 65), and assuming $\nu=1+$ $\gamma_{R_{i}, D}$, we obtain the following equation

$$
\nu\left[B \log _{2}(\nu)-\ln (2)\right]+\mathcal{A}=0,
$$

where $\mathcal{A}=\ln (2)-\left(\frac{\zeta}{\sigma_{n_{D}}^{2}}\right)\left(\ln (2) d_{R_{i}, D}^{-\vartheta}\left|h_{R_{i}, D}\right|^{2}\right)\left(P_{S} d_{S, R_{i}}^{-\vartheta}\right.$ $\left.\left|h_{S, R_{i}}\right|^{2}+\sigma_{n_{R_{i}}}^{2}\right)$.

The solution of the above expression can be expressed as follows

$\nu=\exp \left(\mathcal{W}\left(-\frac{\mathcal{A}}{B} \exp \left(-\frac{1}{B} \log ^{2}(2)\right) * \log (2)\right)+\frac{\log ^{2}(2)}{B}\right)$.

Consequently, the we obtain the following

$$
P_{R_{i}}^{\dagger}=(\nu-1)\left(\frac{\sigma_{n_{D}}^{2}}{d_{R_{i}, D}^{-\vartheta}\left|h_{R_{i}, D}\right|^{2}}\right) .
$$

From 67) and 68, and using (72), we obtain

$$
\phi_{i}^{\dagger}=\frac{r}{B \log _{2}\left(1+\gamma_{S, R_{i}}\right)+\left(\delta_{i} \cdot r\right)} .
$$

Finally, substituting (41) in (68), we find the following

$$
\begin{array}{r}
\theta_{i}^{\dagger}=1-r\left(\frac{1}{B \log _{2}\left(1+\gamma_{S, R_{i}}\right)+\left(\delta_{i} \cdot r\right)}\right. \\
\left.+\frac{1}{B \log _{2}\left(1+\frac{P_{R_{i}}^{\dagger} d_{R_{i}, D}^{-\vartheta}\left|h_{R_{i}, D}\right|^{2}}{\sigma_{n_{D}}^{2}}\right)}\right),
\end{array}
$$

where $P_{R_{i}}^{\dagger}, \phi_{i}^{\dagger}$, and $\theta_{i}^{\dagger}$ are the optimal values obtained for $P_{R_{i}}, \phi_{i}$, and $\theta_{i}$, respectively.

Case VII: $\mu_{1} \neq 0 \Longrightarrow G\left(\theta_{i}, \phi_{i}, P_{R_{i}}\right)=0 ; \mu_{2} \neq 0 \Longrightarrow$ $H\left(\theta_{i}, \phi_{i}, P_{R_{i}}\right)=0 ; \mu_{3}=0 \Longrightarrow I\left(\theta_{i}, \phi_{i}, P_{R_{i}}\right) \neq 0$

The simplified equations for this case can be represented as follows

$$
\begin{gathered}
{\left[\zeta\left(P_{S} d_{S, R_{i}}^{-\vartheta}\left|h_{S, R_{i}}\right|^{2}+\sigma_{n_{R_{i}}}^{2}\right)+P_{R_{i}}\right]+\mu_{1}\left[B \log _{2}\left(1+\gamma_{R_{i}, D}\right)\right]} \\
+\mu_{2}\left[P_{R_{i}}+\zeta\left(P_{S} d_{S, R_{i}}^{-\vartheta}\left|h_{S, R_{i}}\right|^{2}+\sigma_{n_{R_{i}}}^{2}\right)\right]=0, \\
P_{R_{i}}+\mu_{1}\left[B \log _{2}\left(1+\gamma_{R_{i}, D}\right)+\left(B \log _{2}\left(1+\gamma_{S, R_{i}}\right)\right.\right. \\
\left.\left.+\left(\delta_{i} \cdot r\right)\right)\right]+\mu_{2}\left[P_{R_{i}}\right]=0,
\end{gathered}
$$

$\left(1-\left(\theta_{i}+\phi_{i}\right)\right) P_{R_{i}}-\zeta \theta_{i}\left(P_{S} d_{S, R_{i}}^{-\vartheta}\left|h_{S, R_{i}}\right|^{2}+\sigma_{n_{R_{i}}}^{2}\right)-E_{e x t}=0$.

From (77) and (78), and assuming $\mu=1+\gamma_{R_{i}, D}$, we obtain the following equation

$\mu\left(B \log _{2}(\mu)+B \log _{2}\left(1+\gamma_{S, R_{i}}\right)+\left(\delta_{i} \cdot r\right)-\ln (2)\right)+\ln (2)=0$.

Since the solution of 81 is composed of complex values, therefore this case is not acceptable.

Case VIII: $\mu_{1} \neq 0 \Longrightarrow G\left(\theta_{i}, \phi_{i}, P_{R_{i}}\right)=0 ; \mu_{2} \neq 0 \Longrightarrow$ $\left.H \overline{\left(\theta_{i}, \phi_{i}, P_{R_{i}}\right.}\right)=0 ; \mu_{3} \neq 0 \Longrightarrow I\left(\theta_{i}, \phi_{i}, P_{R_{i}}\right)=0$

The equations to be used for computation of $\theta_{i}, \phi_{i}$, and $P_{R_{i}}$ in this case can be written as

$$
\begin{gathered}
{\left[\zeta\left(P_{S} d_{S, R_{i}}^{-\vartheta}\left|h_{S, R_{i}}\right|^{2}+\sigma_{n_{R_{i}}}^{2}\right)+P_{R_{i}}\right]+\mu_{1}\left[B \log _{2}\left(1+\gamma_{R_{i}, D}\right)\right]+} \\
\mu_{2}\left[P_{R_{i}}+\zeta\left(P_{S} d_{S, R_{i}}^{-\vartheta}\left|h_{S, R_{i}}\right|^{2}+\sigma_{n_{R_{i}}}^{2}\right)\right] \\
-\mu_{3}\left[B \log _{2}\left(1+\gamma_{R_{i}, D}\right)\right]=0, \\
P_{R_{i}}+\mu_{1}\left[B \log _{2}\left(1+\gamma_{R_{i}, D}\right)+\left(B \log _{2}\left(1+\gamma_{S, R_{i}}\right)\right.\right. \\
\left.\left.+\left(\delta_{i} \cdot r\right)\right)\right]+\mu_{2}\left[P_{R_{i}}\right]-\mu_{3}\left[B \log _{2}\left(1+\gamma_{R_{i}, D}\right)\right]=0, \\
1+\mu_{1}\left(\frac{\ln (2) d_{R_{i}, D}^{-\vartheta}\left|h_{R_{i}, D}\right|^{2}}{\sigma_{n_{D}}^{2}+P_{R_{i}} d_{R_{i}, D}^{-\vartheta}\left|h_{R_{i}, D}\right|^{2}}\right)+\mu_{2} \\
-\mu_{3}\left(\frac{\ln (2) d_{R_{i}, D}^{-\vartheta}\left|h_{R_{i}, D}\right|^{2}}{\sigma_{n_{D}}^{2}+P_{R_{i}} d_{R_{i}, D}^{-\vartheta}\left|h_{R_{i}, D}\right|^{2}}\right)=0, \\
\left(1-\left(\theta_{i}+\phi_{i}\right)\right) B \log _{2}\left(1+\gamma_{R_{i}, D}\right)-\phi_{i}\left[B \log _{2}\left(1+\gamma_{S, R_{i}}\right)\right. \\
\left.+\left(\delta_{i} \cdot r\right)\right]=0,
\end{gathered}
$$

From (85) and (87), we obtain

$$
\phi_{i}=\frac{r}{B \log _{2}\left(1+\gamma_{S, R_{i}}\right)+\left(\delta_{i} \cdot r\right)} .
$$

Similarly, from (86) and 87, we find

$$
\theta_{i}=\frac{r P_{R_{i}}-E_{e x t} B \log _{2}\left(1+\gamma_{R_{i}, D}\right)}{\zeta\left(P_{S} d_{S, R_{i}}^{-\vartheta}\left|h_{S, R_{i}}\right|^{2}+\sigma_{n_{R_{i}}}^{2}\right)}
$$

Substituting (88) and (89) in (87), and assuming $\eta=1+$ $\gamma_{R_{i}, D}$ we obtain the following equation

$$
\mathcal{A}-B \log _{2}(\eta)\left[\mathcal{B}+\mathcal{C} \eta+\mathcal{D} B \log _{2}(\eta)\right]=0,
$$

where $\mathcal{A}=a \cdot b \cdot r, \mathcal{B}=a \cdot b+a \cdot r \cdot\left(\frac{\sigma_{n_{D}}^{2}}{d_{R_{i}, D}^{-\vartheta}\left|h_{R_{i}, D}\right|^{2}}\right)-b \cdot r$, $\mathcal{C}=-a \cdot r \cdot\left(\frac{\sigma_{n_{D}}^{2}}{d_{R_{i}, D}^{-\vartheta}\left|h_{R_{i}, D}\right|^{2}}\right)$, and $\mathcal{D}=a \cdot E_{\text {ext }}$, where $a=$ $B \log _{2}\left(1+\gamma_{S, R_{i}}\right)+\left(\delta_{i} \cdot r\right)$, and $b=\zeta\left(P_{S} d_{S, R_{i}}^{-\vartheta}\left|h_{S, R_{i}}\right|^{2}+\sigma_{n_{R_{i}}}^{2}\right)$.

Considering $\mathcal{F}(\eta)=\mathcal{A}-B \log _{2}(\eta)\left[\mathcal{B}+\mathcal{C} \eta+\mathcal{D} B \log _{2}(\eta)\right]$, we have a nonlinear equation of the type

$$
\mathcal{F}(\eta)=0 \text {. }
$$

Let us assume that $\eta$ is a simple or one of the multiple roots of (91), and $\eta_{0}$ is an initial point prediction sufficiently near to $\eta$. Using the Taylor's series expansion [cite], we can express the following

$$
\mathcal{F}\left(\eta_{0}\right)+\left(\eta-\eta_{0}\right) \mathcal{F}^{\prime}\left(\eta_{0}\right)+\frac{1}{2}\left(\eta-\eta_{0}\right)^{2} \mathcal{F}^{\prime \prime}\left(\eta_{0}\right)=0
$$


In order to solve the nonlinear equation $\mathcal{F}(\eta)=0$, an alternative equivalence formulation has been used to develop a class of iterative methods. Simplified form of 92 can be re-written as

$$
\mathcal{F}^{\prime \prime}\left(\eta_{0}\right)\left(\eta-\eta_{0}\right)^{2}+2 \mathcal{F}^{\prime}\left(\eta_{0}\right)\left(\eta-\eta_{0}\right)+2 \mathcal{F}\left(\eta_{0}\right)=0
$$

It is clear that the equation in 93 is of the quadratic form. Hence, the corresponding roots can be expressed as

$$
\eta-\eta_{0}=\frac{-\mathcal{F}^{\prime}\left(\eta_{0}\right) \pm \sqrt{\left[\mathcal{F}^{\prime}\left(\eta_{0}\right)\right]^{2}-2 \mathcal{F}^{\prime \prime}\left(\eta_{0}\right) \mathcal{F}\left(\eta_{0}\right)}}{\mathcal{F}^{\prime \prime}\left(\eta_{0}\right)} .
$$

Depending on the sign of proceeding the radical term, the formula in (94) provides the following two possibilities

$$
\begin{aligned}
& \eta=\eta_{0}-\frac{\mathcal{F}^{\prime}\left(\eta_{0}\right)+\sqrt{\left[\mathcal{F}^{\prime}\left(\eta_{0}\right)\right]^{2}-2 \mathcal{F}^{\prime \prime}\left(\eta_{0}\right) \mathcal{F}\left(\eta_{0}\right)}}{\mathcal{F}^{\prime \prime}\left(\eta_{0}\right)} . \\
& \eta=\eta_{0}-\frac{\mathcal{F}^{\prime}\left(\eta_{0}\right)-\sqrt{\left[\mathcal{F}^{\prime}\left(\eta_{0}\right)\right]^{2}-2 \mathcal{F}^{\prime \prime}\left(\eta_{0}\right) \mathcal{F}\left(\eta_{0}\right)}}{\mathcal{F}^{\prime \prime}\left(\eta_{0}\right)} .
\end{aligned}
$$

Using the fixed point formulations in $(95)$ and $(96)$, and in order to maximize the objective in 25 , the following formula for an approximate solution $\eta_{k+1}$ can be used to find the larger root iteratively [35]

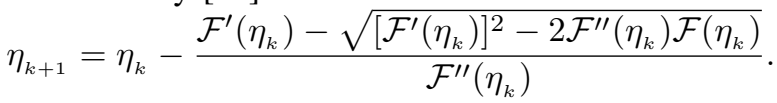

It should be noted that the denominator of (97) is independent of $\mathcal{F}^{\prime}\left(\eta_{k}\right)$ which makes it specially fit to find the largest root of the (91).

Since the nonlinear equation in $(90)$ involves the logarithmic terms, the number of iterations required to find the optimal largest root may be higher for the chosen value of $\eta_{0}$. In that case, Halley's method (2) or the modified Chebyshev's method (39) in [36] may also be used to reduce the number of iterations.

Finally, we obtain the following solutions for this case

$$
\begin{gathered}
P_{R_{i}}^{*}=(\eta-1)\left(\frac{\sigma_{n_{D}}^{2}}{d_{R_{i}, D}^{-\vartheta}\left|h_{R_{i}, D}\right|^{2}}\right), \\
\phi_{i}^{*}=\frac{r \log _{2}\left(1+\gamma_{S, R_{i}}\right)+\left(\delta_{i} \cdot r\right)}{B}, \\
\theta_{i}^{*}=\frac{r P_{R_{i}}^{*}-E_{e x t} B \log _{2}\left(1+\frac{P_{R_{i}}^{*} d_{R_{i}, D}^{-\vartheta}\left|h_{R_{i}, D}\right|^{2}}{\sigma_{n_{D}}^{2}}\right)}{\zeta\left(P_{S} d_{S, R_{i}}^{-\vartheta}\left|h_{S, R_{i}}\right|^{2}+\sigma_{n_{R_{i}}}^{2}\right)},
\end{gathered}
$$

where $P_{R_{i}}^{*}, \phi_{i}^{*}$, and $\theta_{i}^{*}$ are the optimal values obtained for $P_{R_{i}}, \phi_{i}$, and $\theta_{i}$, respectively in this case.

\section{APPENDIX C \\ TS FACTORS' SELECTION FOR UNIFORMITY IN THE ASSUMED CONVENTION}

In this paper, we have investigated two problems for maximization of throughput and stored energy at the relay by using two distinct time-conventions, respectively. This facilitates the reader to independently adopt any of the presented conventions for further analysis in similar directions. Herein, we intend to accomplish the relationship between the two conventions as presented in Fig. 3 and Fig. 4, respectively, in order to impose uniformity in the analysis.

Assuming the TS convention as in Fig. 3, the corresponding conventions for Fig. 4 can be represented in terms of the metrics in Fig. 3 as follows

$$
\theta_{i}=\alpha_{i} \cdot \beta_{i}, \text { and } \phi_{i}=\left(1-\alpha_{i}\right) \cdot \beta_{i},
$$

where $i$ is the index of the chosen relay.

Similarly, if the TS convention as in Fig. 4 is assumed throughout, then the metrics in Fig. 3 can be represented as

$$
\alpha_{i}=\frac{\theta_{i}}{\theta_{i}+\phi_{i}} \text {, and } \beta_{i}=\theta_{i}+\phi_{i} \text {. }
$$

\section{REFERENCES}

[1] S. Gautam, E. Lagunas, S.K. Sharma, S. Chatzinotas, and B Ottersten, "Relay Selection Strategies for SWIPT-Enabled Cooperative Wireless Systems," in 28th Annual IEEE International Symposium on Personal, Indoor and Mobile Radio Communications (PIMRC), October 2017.

[2] N. Janatian, I. Stupia, and L. Vandendorpe, "Optimal resource allocation in ultra-low power fog-computing SWIPT-based networks," in 2018 IEEE Wireless Communications and Networking Conference (WCNC), April 2018, pp. 1-6.

[3] S. Zhou, J. Gong, Z. Zhou, W. Chen, and Z. Niu, "GreenDelivery: proactive content caching and push with energy-harvesting-based small cells," IEEE Commun. Mag., vol. 53, no. 4, pp. 142-149, April 2015.

[4] ERICSSON white paper, "More than 50 Billion Connected Devices ," https://www.ericsson.com/openarticle/mwc-connected-devices_ 1686565587_c 2011, [Online].

[5] D. N. K. Jayakody, J. Thompson, S. Chatzinotas, and S. Durrani, Wireless Information and Power Transfer: A New Paradigm for Green Communications, Springer, 2017.

[6] S. Gautam and P. Ubaidulla, "Simultaneous transmission of information and energy in OFDM systems," in Proc. 18th Wireless Personal Multimedia Communications (WPMC), December 2015.

[7] X. Zhou, R. Zhang, and C. K. Ho, "Wireless Information and Power Transfer in Multiuser OFDM Systems," IEEE Trans. Wireless Commun., vol. 13, no. 4, pp. 2282-2294, April 2014

[8] X. Zhou, R. Zhang, and C. K. Ho, "Wireless Information and Power Transfer: Architecture Design and Rate-Energy Tradeoff," IEEE Trans. Commun., vol. 61, no. 11, pp. 4754-4767, November 2013.

[9] T. D. P. Perera and D. N. K. Jayakody and S. K. Sharma and S. Chatzinotas and J. Li, "Simultaneous Wireless Information and Power Transfer (SWIPT): Recent Advances and Future Challenges," IEEE Commun. Surveys Tuts., 2017.

[10] S. Gautam and P. Ubaidulla, "Simultaneous transmission of information and RF energy in multicarrier systems," in 23rd International Conference on Telecommunications (ICT), May 2016, pp. 1-5.

[11] S. Gautam and P. Ubaidulla, "Relay Selection and Transceiver Design for Joint Wireless Information and Energy Transfer in Cooperative Networks," in 85th Vehicular Technology Conference (VTC), 2017. Spring, June 2017.

[12] Q. Gu, G. Wang, R. Fan, Z. Zhong, K. Yang, and H. Jiang, "Rate-Energy Tradeoff in Simultaneous Wireless Information and Power Transfer over Fading Channels with Uncertain Distribution," IEEE Trans. Veh. Technol., vol. PP, no. 99, pp. 1-1, 2017.

[13] J. Park, B. Clerckx, C. Song, and Y. Wu, "An Analysis of the Optimum Node Density for Simultaneous Wireless Information and Power Transfer in Ad Hoc Networks," IEEE Trans. Veh. Technol., vol. PP, no. 99, pp. 1-1, 2017.

[14] B. Clerckx, "Waveform and Transceiver Design for Simultaneous Wireless Information and Power Transfer," CoRR, vol. abs/1607.05602, 2016.

[15] I. Bang, S. M. Kim, and D. K. Sung, "Adaptive Multiuser Scheduling for Simultaneous Wireless Information and Power Transfer in a Multicell Environment," IEEE Trans. Wireless Commun., vol. 16, no. 11, pp. 7460-7474, Nov. 2017.

[16] S. Mahama, D. K. P. Asiedu, and K. J. Lee, "Simultaneous Wireless Information and Power Transfer for Cooperative Relay Networks With Battery," IEEE Access, vol. 5, pp. 13171-13178, 2017.

[17] A. A. Nasir and X. Zhou and S. Durrani and R. A. Kennedy, "Relaying Protocols for Wireless Energy Harvesting and Information Processing," IEEE Transactions on Wireless Communications, vol. 12, no. 7, pp. 3622-3636, July 2013.

[18] J. Huang, C. C. Xing, and C. Wang, "Simultaneous Wireless Information and Power Transfer: Technologies, Applications, and Research Challenges," IEEE Commun. Mag., vol. 55, no. 11, pp. 26-32, Nov. 2017. 
[19] S. Borst, V. Gupta, and A. Walid, "Distributed Caching Algorithms for Content Distribution Networks," in Proc. IEEE Int. Conf. Comput. Commun., Mar. 2010, pp. 1-9.

[20] M. A. Maddah-Ali and U. Niesen, "Fundamental Limits of Caching," IEEE Trans. Inf. Theory, vol. 60, no. 5, pp. 2856-2867, May 2014.

[21] T. X. Vu, S. Chatzinotas, B. Ottersten, and T. Q. Duong, "Energy Minimization for Cache-assisted Content Delivery Networks with Wireless Backhaul," IEEE Wireless Commun. Lett., vol. 7, no. 3, pp. 332 - 335, Jun 2018.

[22] T. X. Vu, S. Chatzinotas, and B. Ottersten, "Edge-Caching Wireless Networks: Performance analysis and optimization," IEEE Trans. Wireless Commun., vol. 17, no. 7, pp. 2827 - 2839, Apr 2018.

[23] S. Gautam and T. X. Vu and S. Chatzinotas and B. Ottersten, "Joint wireless information and energy transfer in cache-assisted relaying systems," in IEEE Wireless Communications and Networking Conference (WCNC), April 2018, pp. 1-6.

[24] S. Vuppala, T. X. Vu, S. Gautam, S. Chatzinotas, and B. Ottersten, "Cache-Aided Millimeter Wave Ad-Hoc Networks with ContentionBased Content Delivery," IEEE Trans. Commun., vol. 66, no. 8, pp. 3540 - 3554, Aug 2018.

[25] A. Kumar and W. Saad, "On the tradeoff between energy harvesting and caching in wireless networks," in 2015 IEEE International Conference on Communication Workshop (ICCW), Jun. 2015, pp. 1976-1981.

[26] D. Niyato, D. I. Kim, P. Wang, and L. Song, "A novel caching mechanism for Internet of Things (IoT) sensing service with energy harvesting," in Proc. IEEE Int. Conf. Commun., May 2016, pp. 1-6.

[27] S. Zhang, N. Zhang, X. Fang, P. Yang, and X. S. Shen, "Cost-effective vehicular network planning with cache-enabled green roadside units," in Proc. IEEE Int. Conf. Commun., May 2017, pp. 1-6.

[28] I. Krikidis and S. Timotheou and S. Nikolaou and G. Zheng and D. W. K. Ng and R. Schober, "Simultaneous wireless information and power transfer in modern communication systems," IEEE Commun. Mag., vol 52, no. 11, pp. 104-110, Nov 2014.

[29] L. Xiang and D. W. K. Ng and T. Islam and R. Schober and V. W. S. Wong and J. Wang, "Cross-Layer Optimization of Fast Video Delivery in Cache- and Buffer-Enabled Relaying Networks," IEEE Trans. Veh. Technol., vol. 66, no. 12, pp. 11366-11382, Dec 2017.

[30] S. Lohani, R. A. Loodaricheh, E. Hossain, and V. K. Bhargava, "On Multiuser Resource Allocation in Relay-Based Wireless-Powered Uplink Cellular Networks," IEEE Trans. Wireless Commun., vol. 15, no. 3, pp. 1851-1865, March 2016

[31] F. Benkhelifa and M. S. Alouini, "Precoding Design of MIMO Amplifyand-Forward Communication System With an Energy Harvesting Relay and Possibly Imperfect CSI," IEEE Access, vol. 5, pp. 578-594, 2017.

[32] Boyd, Stephen and Vandenberghe, Lieven, Convex optimization, Cambridge university press, 2004

[33] Robert M. Corless, Gaston H. Gonnet, David E. G. Hare, David J. Jeffrey, and Donald E. Knuth, "On the LambertW function," Advances in Computational mathematics, vol. 5, no. 1, pp. 329-359, 1996.

[34] U. Muncuk and K. Alemdar and J. D. Sarode and K. R. Chowdhury, "Multi-band Ambient RF Energy Harvesting Circuit Design for Enabling Battery-less Sensors and IoTs," http://krc.coe.neu.edu/sites/krc. coe.neu.edu/files/papers/IOTJournal18\%20.pdf accepted to IEEE Internet Things J., Feb 2018, [Online].

[35] Z. Hong-bin, "A Novel Method Finding Multiple Roots of Nonlinear Equations," in 2009 Fifth International Conference on Natural Computation, Aug 2009, vol. 6, pp. 299-302.

[36] Beny Neta, "New third order nonlinear solvers for multiple roots," Applied Mathematics and Computation, vol. 202, no. 1, pp. 162-170, 2008.

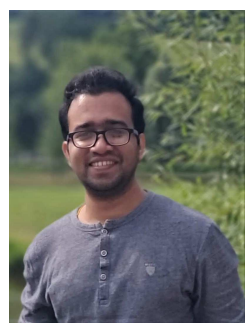

Sumit Gautam (S'14) received the B.Tech. degree (Hons.) in electronics and communication engineering from the LNM Institute of Information Technology (Deemed University), Jaipur, Rajasthan, India in 2013 and the MS degree in electronics and communication engineering by research from the International Institute of Information Technology (Deemed University), Hyderabad, Telangana, India in 2017. He is currently pursuing the Ph.D. degree in computer science from the Interdisciplinary Centre for Security, Reliability, and Trust (SnT), University of Luxembourg, Luxembourg. His research interests include simultaneous wireless transmission of information and energy (Wi-TIE), caching, optimization methods, and cooperative communications.

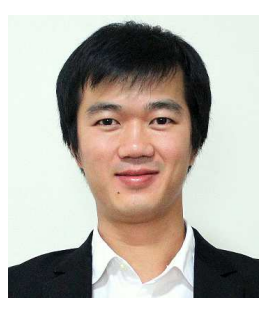

Thang X. Vu (S'11-M'15) was born in Hai Duong, Vietnam. He received the B.S. and the M.Sc., both in Electronics and Telecommunications Engineering, from the VNU University of Engineering and Technology, Vietnam, in 2007 and 2009, respectively, and the Ph.D. in Electrical Engineering from the University Paris-Sud, France, in 2014

From 2007 to 2009, he was with the Department of Electronics and Telecommunications, VNU University of Engineering and Technology, Vietnam as a research assistant. In 2010, he received the Allocation de Recherche fellowship to study Ph.D. in France. From September 2010 to May 2014, he was with the Laboratory of Signals and Systems (LSS), a joint laboratory of CNRS, CentraleSupelec and University Paris-Sud XI, France. From July 2014 to January 2016, he was postdoctoral researcher with the Information Systems Technology and Design (ISTD) pillar, Singapore University of Technology and Design (SUTD), Singapore. Currently, he is research associate at Interdisciplinary Centre for Security, Reliability and Trust (SnT), University of Luxembourg. His research interests are in the field of wireless communications, with particular interests of cache-assisted $5 \mathrm{G}$, machine learning for communications, cloud radio access networks, and resources allocation and optimization.

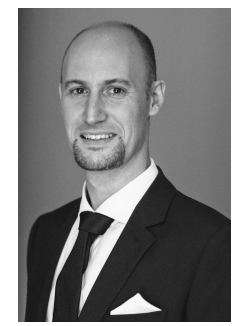

Symeon Chatzinotas (S'06-M'09-SM'13) is currently the Deputy Head of the SIGCOM Research Group, Interdisciplinary Centre for Security, Reliability, and Trust, University of Luxembourg, Luxembourg and Visiting Professor at the University of Parma, Italy. He received the M.Eng. degree in telecommunications from the Aristotle University of Thessaloniki, Thessaloniki, Greece, in 2003, and the M.Sc. and Ph.D. degrees in electronic engineering from the University of Surrey, Surrey, U.K., in 2006 and 2009, respectively. He was involved in numerous Research and Development projects for the Institute of Informatics Telecommunications, National Center for Scientific Research Demokritos, the Institute of Telematics and Informatics, Center of Research and Technology Hellas, and the Mobile Communications Research Group, Center of Communication Systems Research, University of Surrey. He has over 300 publications, 3000 citations, and an H-Index of 28 according to Google Scholar. His research interests include multiuser information theory, co-operative/cognitive communications, and wireless networks optimization. He was a co-recipient of the 2014 IEEE Distinguished Contributions to Satellite Communications Award, the CROWNCOM 2015 Best Paper Award and the 2018 EURASIC JWCN Best Paper Award.

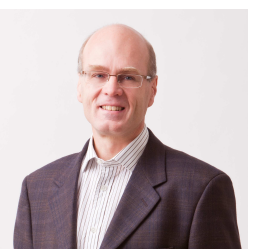

Björn Ottersten (''87-M'89-SM'99-F'04) was born in Stockholm, Sweden, in 1961. He received the M.S. degree in electrical engineering and applied physics from Linköping University, Linköping, Sweden, in 1986, and the Ph.D. degree in electrical engineering from Stanford University, Stanford, CA, USA, in 1990. He has held research positions with the Department of Electrical Engineering, Linköping University, the Information Systems Laboratory, Stanford University, the Katholieke Universiteit Leuven, Leuven, Belgium, and the University of Luxembourg, Luxembourg. From 1996 to 1997, he was the Director of Research with ArrayComm, Inc., a start-up in San Jose, CA, USA, based on his patented technology. In 1991, he was appointed a Professor of signal processing with the Royal Institute of Technology (KTH), Stockholm, Sweden. From 1992 to 2004, he was the Head of the Department for Signals, Sensors, and Systems, KTH, and from 2004 to 2008, he was the Dean of the School of Electrical Engineering, KTH. He is currently the Director for the Interdisciplinary Centre for Security, Reliability and Trust, University of Luxembourg.

He was a recipient of the IEEE Signal Processing Society Technical Achievement Award in 2011 and the European Research Council advanced research grant twice, in 2009-2013 and in 2017-2022. He has co-authored journal papers that received the IEEE Signal Processing Society Best Paper Award in 1993, 2001, 2006, and 2013, and seven IEEE conference papers best paper awards. He has served as an Associate Editor for the IEEE TRANSACTIONS ON SIGNAL PROCESSING and the Editorial Board of the IEEE Signal Processing Magazine. He is currently a member of the editorial boards of EURASIP Signal Processing Journal, EURASIP Journal of Advances Signal Processing and Foundations and Trends of Signal Processing. $\mathrm{He}$ is a fellow of EURASIP. 